\title{
The Products of Regularly Solvable Operators with Their Spectra in Direct Sum Spaces
}

\author{
Sobhy El-Sayed Ibrahim \\ Department of Mathematics, Faculty of Science, Benha University, Benha, Egypt \\ Email: sobhyelsayed_55@hotmail.com
}

Received January 21, 2013; revised March 14, 2013; accepted April 29, 2013

Copyright (C) 2013 Sobhy El-Sayed Ibrahim. This is an open access article distributed under the Creative Commons Attribution License, which permits unrestricted use, distribution, and reproduction in any medium, provided the original work is properly cited.

\begin{abstract}
In this paper, we consider the general quasi-differential expressions $\tau_{1}, \tau_{2}, \cdots, \tau_{n}$ each of order $n$ with complex coefficients and their formal adjoints on the interval $(a, b)$. It is shown in direct sum spaces $L_{w}^{2}\left(I_{p}\right), p=1,2, \cdots, N$ of functions defined on each of the separate intervals with the cases of one and two singular end-points and when all solutions of the equation $\left[\prod_{j=1}^{n} \tau_{j}-\lambda w\right] u=0$ and its adjoint $\left[\prod_{j=1}^{n} \tau_{j}^{+}-\bar{\lambda} w\right] v=0$ are in $L_{w}^{2}(a, b)$ (the limit circle case) that all well-posed extensions of the minimal operator $T_{0}\left(\tau_{1}, \tau_{2}, \cdots, \tau_{n}\right)$ have resolvents which are HilbertSchmidt integral operators and consequently have a wholly discrete spectrum. This implies that all the regularly solvable operators have all the standard essential spectra to be empty. These results extend those of formally symmetric expression $\tau$ studied in [1-10] and those of general quasi-differential expressions $\tau$ in [11-19].
\end{abstract}

Keywords: Product of Quasi-Differential Expressions; Regular and Singular Endpoints; Regularly Solvable Operators; Essential Spectra; Hilbert-Schmidt Integral Operators

\section{Introduction}

The operators which fulfill the role that the self-adjoint and maximal symmetric operators play in the case of a formally symmetric expression $\tau$ are those which are regularly solvable with respect to the minimal operators $T_{0}(\tau)$ and $T_{0}\left(\tau^{+}\right)$generated by a general ordinary quasi-differential expression $\tau$ and its formal adjoint $\tau^{+}$respectively, the minimal operators $T_{0}(\tau)$ and $T_{0}\left(\tau^{+}\right)$form an adjoint pair of closed, densely-defined operators in the underlying $L_{w}^{2}$-space, that is

$T_{0}(\tau) \subset\left[T_{0}\left(\tau^{+}\right)\right]^{*}$. Such an operator $S$ satisfies

$T_{0}(\tau) \subset S \subset\left[T_{0}\left(\tau^{+}\right)\right]^{*}$ and for some $\lambda \in \mathbb{C}$, the operator $(S-\lambda I)$ is a Fredholm operator of zero index, this means that $S$ has the desirable Fredholm property that the equation $(S-\lambda I) u=f$ has a solution if and only if $f$ is orthogonal to the solution space of $(S-\lambda I) u=0$ and furthermore the solution space of $(S-\lambda I) u=0$ and $\left(S^{*}-\bar{\lambda} I\right) v=0$ have the same finite dimension. This notion was originally due to Visik [20].
Akhiezer and Glazman [1] and Naimark [2] are showed that the self-adjoint extension $S$ of the minimal operator $T_{0}(\tau)$ generated by a formally symmetric differential expression $\tau$ with maximal deficiency indices have resolvents which are Hilbert-Schmidt integral operators and consequently have a wholly discrete spectrum. In $[15,16,18,19]$ Ibrahim extend their results for general ordinary quasi-differential expression $\tau$ of $n$-th order with complex coefficients in the singular case.

In $[3,8]$ Everitt and Zettl considered the problem of integrable square solutions of products of differential expressions $\tau_{1}, \tau_{2}, \cdots, \tau_{n}$ and investigate the relationship between the deficiency indices of general symmetric differential expressions $\tau_{1}, \tau_{2}, \cdots, \tau_{n}$ and those of the product expression $\prod_{j=1}^{n} \tau_{j}$ and in [17] Ibrahim considered the problem of the point spectra and regularity fields for products of a general quasi-differential operators.

Our objective in this paper is a generalization of the results in $[6,7,15,16,18,19]$ for the product quasi-differential operators $\prod_{j=1}^{n} T_{0}\left(\tau_{j}\right), \prod_{j=1}^{n} T_{0}\left(\tau_{j}^{+}\right)$and their spectra in direct sum spaces $L_{w}^{2}\left(I_{p}\right), p=1,2, \cdots, N$ of func- 
tions defined on each of the separate intervals with the cases of one and two singular end-points and when all solutions of the product equations $\left[\prod_{j=1}^{n} \tau_{j p}-\lambda w\right] u=0$ and $\left[\prod_{j=1}^{n} \tau_{j p}^{+}-\bar{\lambda} w\right] v=0$ are in $L_{w}^{2}\left(I_{p}\right)$ for some (and hence all $\lambda \in C$. The end-points of $I_{p}$ assumed to be regular or may be singular.

We deal throughout this paper with a quasi-differential expression $\tau$ of arbitrary order $n$ defined by ShinZettl matrices [14], and the minimal operator $T_{0}(\tau)$ generated by $w^{-1} \tau[$.$] in L_{w}^{2}(I)$, where $w$ is a positive weight function on the underlying interval $I$. The endpoints $a$ and $b$ of $I$ may be regular or singular endpoints.

\section{Notation and Preliminaries}

We begin with a brief survey of adjoint pairs of operators and their associated regularly solvable operators; a full treatment may be found in [2,7,11, Chapter III], [12, $15,16,18]$. The domain and range of a linear operator $T$ acting in a Hilbert space $H$ will be denoted by $D(T)$ and $R(T)$ respectively and $N(T)$ will denote its null space. The nullity of $T$, written $\operatorname{nul}(T)$, is the dimension of $N(T)$ and the deficiency of $T$, written $\operatorname{def}(T)$, is the co-dimension of $R(T)$ in $H$; thus if $T$ is densely defined and $R(T)$ is closed, then $\operatorname{def}(T)=\operatorname{null}\left(T^{*}\right)$. The Fredholm domain of $T$ is (in the notation of [13]) the open subset $\Delta_{3}(T)$ of $\mathbb{C}$ consisting of those values of $\lambda \in \mathbb{C}$ which are such that $(T-\lambda I)$ is a Fredholm operator, where $I$ is the identity operator in $H$. Thus $\lambda \in \Delta_{3}(T)$ if and only if $(T-\lambda I)$ has closed range and finite nullity and deficiency. The index of $(T-\lambda I)$ is the number ind $(T-\lambda I)=\operatorname{nul}(T-\lambda I)-\operatorname{def}(T-\lambda I)$, this being defined for $\lambda \in \Delta_{3}(T)$.

Two closed densely defined operators $A$ and $B$ acting in a Hilbert space $H$ are said to form an adjoint pair if $A \subset B^{*}$ and, consequently, $B \subset A^{*}$; equivalently, $(A x, y)=(x, B y)$ for all $x \in D(A)$ and $y \in D(B)$, where $(.,$.$) denotes the inner-product on H$.

Definition 2.1: The field of regularity $\Pi(A)$ of $A$ is the set of all $\lambda \in \mathbb{C}$ for which there exists a positive constant $K(\lambda)$ such that

$$
\|(A-\lambda I) x\| \geq K(\lambda)\|x\| \text { for all } x \in D(A),
$$

or, equivalently, on using the Closed Graph Theorem, $\operatorname{nul}(A-\lambda I)=0$ and $R(A-\lambda I)$ is closed.

The joint field of regularity $\Pi(A, B)$ of $A$ and $B$ is the set of $\lambda \in \mathbb{C}$ which are such that $\lambda \in \Pi(A)$, $\bar{\lambda} \in \Pi(B)$ and both $\operatorname{def}(A-\lambda I)$ and $\operatorname{def}(B-\bar{\lambda} I)$ are finite. An adjoint pair $A$ and $B$ is said to be compatible if $\Pi(A, B) \neq \phi$.

Definition 2.2: A closed operator $S$ in $H$ is said to be regularly solvable with respect to the compatible adjoint pair of $A$ and $B$ if $A \subset S \subset B^{*}$ and $\Pi(A, B) \cap \Delta_{4}(S) \neq \phi$, where $\Delta_{4}(S)=\left\{\lambda: \lambda \in \Delta_{3}(S)\right.$,ind $\left.(S-\lambda I)=0\right\}$.

Definition 2.3: The resolvent set $\rho(S)$ of a closed operator $S$ in $H$ consists of the complex numbers $\lambda$ for which $(S-\lambda I)^{-1}$ exists, is defined on $H$ and is bounded. The complement of $\rho(S)$ in $\mathbb{C}$ is called the spectrum of $S$ and written $\sigma(S)$. The point spectrum $\sigma_{p}(S)$, continuous spectrum $\sigma_{c}(S)$ and residual spectrum $\sigma_{r}(S)$ are the following subsets of $\sigma(S)$ (see $[11,15]$, and [16]).

$\sigma_{p}(S)=\{\lambda \in \sigma(S):(S-\lambda I)$ is not injective $\}$, i.e., the set of eigenvalues of $S$;

$$
\begin{gathered}
\sigma_{c}(S)=\{\lambda \in \sigma(S):(S-\lambda I) \text { is injective, } \\
R(S-\lambda I) \subsetneq \overline{R(S-\lambda I)}=H\} ;
\end{gathered}
$$

$\sigma_{r}(S)$

$=\{\lambda \in \sigma(S):(S-\lambda I)$ is injective, $\overline{R(S-\lambda I)} \neq H\}$.

For a closed operator $S$ we have,

$$
\sigma(S)=\sigma_{p}(S) \cup \sigma_{c}(S) \cup \sigma_{r}(S) .
$$

An important subset of the spectrum of a closed densely defined operator $S$ in $H$ is the so-called essential spectrum. The various essential spectra of $S$ are defined as in [11, Chapter 9] to be the sets:

$$
\sigma_{e k}(S)=\mathbb{C} \backslash \Delta_{k}(S),(k=1,2,3,4,5),
$$

where $\Delta_{3}(S)$ and $\Delta_{4}(S)$ have been defined earlier.

Definition 2.4: For two closed densely defined operators $A$ and $B$ acting in $H$, if $A \subset S \subset B^{*}$ and the resolvent set $\rho(S)$ of $S$ is nonempty (see $[11,12]$ ), $S$ is said to be well-posed with respect to $A$ and $B$.

Note that, if $A \subset S \subset B^{*}$ and $\lambda \in \rho(S)$ then $\lambda \in \Pi(A)$ and $\bar{\lambda} \in \rho\left(S^{*}\right) \subset \Pi(B)$ so that if $\operatorname{def}(A-\lambda I)$ and $\operatorname{def}(B-\bar{\lambda} I)$ are finite, then $A$ and $B$ are compatible, in this case $S$ is regularly solvable with respect to $A$ and $B$. The terminology "regularly solvable" comes from Visik's paper [20], while the notion of "well posed" was introduced by Zhikhar in his work on $J$-self adjoint operators in [21].

Given two operators $A$ and $B$ both acting in a Hilbert space $H$, we wish to consider the product operator $A B$. This is defined as follows

$$
\begin{aligned}
& D(A B)=\{x \in D(B) \mid B x \in D(A)\} \\
& \text { and }(A B) x=A(B x) \text { for all } x \in D(A B) .
\end{aligned}
$$

It may happen in general that $D(A B)$ contains only the null element of $H$. However, in the case of many differential operators the domains of the product will be 
dense in $H$.

The next result gives conditions under which the deficiency of a product is the sum of the deficiencies of the factors. It is a generalization of that in [3, Theorem A] and [8].

Lemma 2.5 (cf. [17, Lemma 2.3]). Let $A$ and $B$ be closed operators with dense domains in a Hilbert space $H$. Suppose that $\lambda \in \Pi(A, B)$. Then $A B$ is a closed operator with dense domain and

$$
\operatorname{def}(A B-\lambda I)=\operatorname{def}(A-\lambda I)+\operatorname{def}(B-\bar{\lambda} I) .
$$

Evidently Lemma 2.5 extends to the product of any finite number of operators $A_{1}, A_{2}, \cdots, A_{n}$.

\section{Quasi-Differential Expressions in Direct Sum Spaces}

The quasi-differential expressions are defined in terms of a Shin-Zettl matrix $F_{p}$ on an interval $I_{p}$. The set $Z_{n}\left(I_{p}\right)$ of Shin-Zettl matrices on $I_{p}$ consists of $n \times n$-matrices $F_{p}=\left\{f_{r s}^{p}\right\}, p=1,2, \cdots, N$, whose entries are complex-valued functions on $I_{p}$ which satisfy the following conditions:

$$
\begin{aligned}
& f_{r s}^{p} \in L_{l o c}^{2}\left(I_{p}\right),(1 \leq r, s \leq n, n \geq 2) \\
& f_{r, r+1}^{p} \neq 0, \text { a.e., on } I_{p}(1 \leq r \leq n-1) \\
& f_{r s}^{p}=0, \text { a.e., on } I_{p},(2 \leq r+1<s \leq n), p=1,2, \cdots, N .
\end{aligned}
$$

For $F_{p} \in Z_{n}\left(I_{p}\right)$, the quasi-derivatives associated with $F_{p}$ are defined by:

$$
\begin{aligned}
& y^{[0]}:=y, \\
& y^{[r]}:=\left(f_{r, r+1}^{p}\right)^{-1}\left\{\left(y^{[r-1]}\right)^{\prime}-\sum_{s=1}^{r} f_{r s}^{p} y^{[s-1]}\right\}, \quad(1 \leq r \leq n-1), \\
& y^{[n]}:=\left\{\left(y^{[n-1]}\right)^{\prime}-\sum_{s=1}^{n} f_{r s}^{p} y^{[n-1]}\right\},
\end{aligned}
$$

where the prime ' denotes differentiation.

The quasi-differential expression $\tau_{p}$ associated with $F_{p}$ is given by:

$$
\tau_{p}[.]:=i^{n} y^{[n]},(n \geq 2),
$$

this being defined on the set:

$$
\begin{aligned}
& V\left(\tau_{p}\right):=\left\{y: y^{[r-1]} \in A C_{\text {loc }}\left(I_{p}\right), r=1,2, \cdots, n\right\}, \\
& p=1,2, \cdots, N
\end{aligned}
$$

where $A C_{l o c}\left(I_{p}\right)$, denotes the set of functions which are absolutely continuous on every compact subinterval of $I_{p}$.

The formal adjoint $\tau_{p}^{+}$of $\tau_{p}$ is defined by the matrix $F_{p}^{+}$given by:

$$
\tau_{p}^{+}[.]:=i^{n} y_{+}^{[n]}, \text { for all } y \in V\left(\tau_{p}^{+}\right),
$$

$$
\begin{aligned}
& V\left(\tau_{p}^{+}\right):=\left\{y: y_{+}^{[r-1]} \in A C_{l o c}\left(I_{p}\right), r=1,2, \cdots, n\right\}, \\
& p=1,2, \cdots, N
\end{aligned}
$$

where $y_{+}^{[r-1]}$, the quasi-derivatives associated with the matrix $F_{p}^{+}$in $Z_{n}\left(I_{p}\right)$,

$$
F_{p}^{+}=\left(f_{r s}^{p}\right)^{+}=(-1)^{r+s+1} \overline{f_{n-s+1, n-r+1}^{p}} \text {, for each } r \text { and } s .
$$

Note that: $\left(F_{p}^{+}\right)^{+}=F_{p}$ and so $\left(\tau_{p}^{+}\right)^{+}=\tau_{p}$. We refer to $[3,9]$ and [17-20] for a full account of the above and subsequent results on quasi-differential expressions.

For $u \in V\left(\tau_{p}\right), v \in V\left(\tau_{p}^{+}\right)$and $\alpha, \beta \in I_{p}$, we have Green's formula,

$$
\begin{aligned}
& \int_{a_{p}}^{b_{p}}\left\{\bar{v} \tau_{p}[u]-u \overline{\tau_{p}^{+}[v]}\right\} \mathrm{d} x \\
& =[u, v]\left(b_{p}\right)-[u, v]\left(a_{p}\right), \quad p=1,2, \cdots, N,
\end{aligned}
$$

where,

$$
\begin{aligned}
{[u, v](x) } & =i^{n}\left(\sum_{r=0}^{n-1}(-1)^{r+s+1} u^{[r]}(x) \overline{v_{+}^{[n-r-1]}}(x)\right) \\
& =(-i)^{n}\left(u, u^{[1]}, \cdots, u^{[n-1]}\right) \times J_{n \times n}\left(\begin{array}{c}
\bar{v} \\
\vdots \\
\bar{v}_{+}^{[n-1]}
\end{array}\right)(x) ;
\end{aligned}
$$

see [4,9] and [14-18]. Let the interval $I_{p}$ have endpoints $a_{p}, b_{p}\left(-\infty \leq a_{p}<b_{p} \leq \infty\right)$, and let $w_{p}: I_{p} \rightarrow \mathbb{R}$ be a non-negative weight function with $w_{p} \in L_{\text {loc }}^{1}\left(I_{p}\right)$ and $w_{p}>0$ (for almost all $x \in I_{p}$ ). Then $H_{p}=L_{w_{p}}^{2}\left(I_{p}\right)$ denotes the Hilbert function space of equivalence classes of Lebesgue measurable functions such that $\int_{I_{p}} w_{p}|f|^{2}<\infty$; the inner-product is defined
by:

$$
\begin{aligned}
& (f, g)_{p}:=\int_{I_{p}} w_{p} f(x) \overline{g(x)} \mathrm{d} x \\
& \left(f, g \in L_{w_{p}}^{2}\left(I_{p}\right) \cdot p=1,2, \cdots, N\right) .
\end{aligned}
$$

The equation

$$
\tau_{p}[u]-\lambda w_{p} u=0(\lambda \in \mathbb{C}) \text { on } I_{p}, p=1,2, \cdots, N,
$$

is said to be regular at the left end-point $a_{p} \in \mathbb{R}$, if for all $X \in\left(a_{p}, b_{p}\right)$,

$$
\begin{aligned}
& a_{p} \in \mathbb{R}, w_{p}, f_{r s}^{p} \in L^{1}\left(a_{p}, X\right), \\
& (r, s=1,2, \cdots, n ; p=1,2, \cdots, N) .
\end{aligned}
$$

Otherwise (3.9) is said to be singular at $a_{p}$. If (3.9) is regular at both end-points, then it is said to be regular; in this case we have,

$$
\begin{aligned}
& a_{p}, b_{p} \in \mathbb{R}, w_{p}, f_{r s}^{p} \in L^{1}\left(a_{p}, b_{p}\right), \\
& (r, s=1,2, \cdots, n ; p=1,2, \cdots, N) .
\end{aligned}
$$


We shall be concerned with the case when $a_{p}$ is a regular end-point of (3.9), the end-point $b_{p}$ being allowed to be either regular or singular. Note that, in view of (3.5), an end-point of $I_{p}$ is regular for (3.9), if and only if it is regular for the equation,

$$
\tau_{p}^{+}[v]-\bar{\lambda} w_{p} v=0(\lambda \in \mathbb{C}) \text { on } I_{p}, p=1,2, \cdots, N,
$$

Note that, at a regular end-point $a_{p}$, say, $u^{[r-1]}\left(a_{p}\right)\left(v_{+}^{[r-1]}\left(a_{p}\right)\right), r=1,2, \cdots, n$ is defined for all $u \in V\left(\tau_{p}\right) \quad\left(v \in V\left(\tau_{p}^{+}\right)\right)$. Set:

$D\left(\tau_{p}\right):=\left\{u: u \in V\left(\tau_{p}\right), u\right.$ and $\left.w_{p}^{-1} \tau_{p}[u] \in L_{w_{p}}^{2}\left(a_{p}, b_{p}\right)\right\}$, $p=1,2, \cdots, N$

$D\left(\tau_{p}^{+}\right):=\left\{v: v \in V\left(\tau_{p}^{+}\right), v\right.$ and $\left.w_{p}^{-1} \tau_{p}^{+}[v] \in L_{w_{p}}^{2}\left(a_{p}, b_{p}\right)\right\}$, $p=1,2, \cdots, N$.

The subspaces $D\left(\tau_{p}\right)$ and $D\left(\tau_{p}^{+}\right)$of $L_{w_{p}}^{2}\left(a_{p}, b_{p}\right)$ are domains of the so-called maximal operators $T\left(\tau_{p}\right)$ and $T\left(\tau_{p}^{+}\right)$respectively, defined by:

$T\left(\tau_{p}\right) u:=w_{p}^{-1} \tau_{p}[u], \quad\left(u \in D\left(\tau_{p}\right)\right)$ and

$T\left(\tau_{p}^{+}\right) v:=w_{p}^{-1} \tau_{p}^{+}[v], \quad\left(v \in D\left(\tau_{p}^{+}\right)\right)$.

For the regular problem the minimal operators $T_{0}\left(\tau_{p}\right)$ and $T_{0}\left(\tau_{p}^{+}\right), p=1,2, \cdots, N$ are the restrictions of $w_{p}^{-1} \tau_{p}[u]$ and $w_{p}^{-1} \tau_{p}^{+}[v]$ to the subspaces:

$$
\begin{aligned}
& D_{0}\left(\tau_{p}\right):= \\
& \left\{u: u \in D\left(\tau_{p}\right), u^{[r-1]}\left(a_{p}\right)=u^{[r-1]}\left(b_{p}\right), p=1,2, \cdots, N\right\} \\
& D_{0}\left(\tau_{p}^{+}\right):= \\
& \left\{v: v \in D\left(\tau_{p}^{+}\right), v_{+}^{[r-1]}\left(a_{p}\right)=v_{+}^{[r-1]}\left(b_{p}\right), p=1,2, \cdots, N\right\},
\end{aligned}
$$

respectively. The subspaces $D_{0}\left(\tau_{p}\right)$ and $D_{0}\left(\tau_{p}^{+}\right)$are dense in $L_{w_{p}}^{2}\left(a_{p}, b_{p}\right)$ and $T_{0}\left(\tau_{p}\right)$ and $T_{0}\left(\tau_{p}^{+}\right)$are closed operators (see $[2,5,9$, Section 3], $[11,13,16]$ ).

In the singular problem we first introduce the operators $T_{0}^{\prime}\left(\tau_{p}\right)$ and $T_{0}^{\prime}\left(\tau_{p}^{+}\right) ; T_{0}^{\prime}\left(\tau_{p}\right)$ being the restriction of $w_{p}^{-1} \tau_{p}[$.$] to the subspace:$

$$
\begin{aligned}
& D_{0}^{\prime}\left(\tau_{p}\right) \\
:= & \left\{u: u \in D\left(\tau_{p}\right), \operatorname{supp}(u) \subset\left(a_{p}, b_{p}\right), p=1,2, \cdots, N\right\}
\end{aligned}
$$

and with $T_{0}^{\prime}\left(\tau_{p}^{+}\right)$defined similarly. These operators are densely-defined and closable in $L_{w_{p}}^{2}\left(a_{p}, b_{p}\right)$; and we define the minimal operators $T_{0}\left(\tau_{p}\right)$ and $T_{0}\left(\tau_{p}^{+}\right)$to be their respective closures (see $[12,13,16,19])$. We denote the domains of $T_{0}\left(\tau_{p}\right)$ and $T_{0}\left(\tau_{p}^{+}\right)$by $D_{0}\left(\tau_{p}\right)$ and $D_{0}\left(\tau_{p}^{p}\right)$ respectively. It can be shown that:

$$
\begin{aligned}
& u \in D_{0}\left(\tau_{p}\right) \Rightarrow u^{[r-1]}\left(a_{p}\right)=0, \\
& (r=1,2, \cdots, n ; p=1,2, \cdots, N), \\
& v \in D_{0}\left(\tau_{p}^{+}\right) \Rightarrow v_{+}^{[r-1]}\left(a_{p}\right)=0, \\
& (r=1,2, \cdots, n ; p=1,2, \cdots, N)
\end{aligned}
$$

because we are assuming that $a_{p}$ is a regular end-point. Moreover, in both regular and singular problems, we have

$$
T_{0}^{*}\left(\tau_{p}\right)=T\left(\tau_{p}^{+}\right), T_{0}^{*}\left(\tau_{p}\right)=T_{0}\left(\tau_{p}^{+}\right), p=1,2, \cdots, N ;
$$

see [8, Section 5] in the case when $\tau_{p}=\tau_{p}^{+}$and compare with treatment in [11, Section III.10.3] and [16] in general case.

In the case of two singular end-points, the problem on $\left(a_{p}, b_{p}\right)$ is effectively reduced to the problems with one singular end-point on the intervals $\left(a_{p}, c_{p}\right]$ and $\left[c_{p}, b_{p}\right)$, where $c_{p} \in\left(a_{p}, b_{p}\right)$. We denote by $T\left(\tau_{p} ; a_{p}\right)$ and $T\left(\tau_{p} ; b_{p}\right)$ the maximal operators with domains $D\left(\tau_{p} ; a_{p}\right)$ and $D\left(\tau_{p} ; b_{p}\right)$ and denote $T_{0}\left(\tau_{p} ; a_{p}\right)$ and $T_{0}\left(\tau_{p} ; b_{p}\right)$ the closures of the operators $T_{0}^{\prime}\left(\tau_{p} ; a_{p}\right)$ and $T_{0}^{\prime}\left(\tau_{p} ; b_{p}\right)$ defined by:

$$
\begin{aligned}
& D_{0}^{\prime}\left(\tau_{p} ; .\right) \\
:= & \left\{u: u \in D\left(\tau_{p} ; .\right), \operatorname{supp}(u) \subset\left(a_{p}, b_{p}\right), p=1,2, \cdots, N\right\}
\end{aligned}
$$

on the intervals $\left(a_{p}, c_{p}\right]$ and $\left[c_{p}, b_{p}\right)$ respectively, see $\left([2,5,9,11,13]\right.$ and [16]). Let $\tilde{T}_{0}^{\prime}\left(\tau_{p}\right), p=1,2, \cdots, N$, be the orthogonal sum as:

$$
\begin{aligned}
& \tilde{T}_{0}^{\prime}\left(\tau_{p}\right)=T_{0}^{\prime}\left(\tau_{p} ; a_{p}\right) \oplus T_{0}^{\prime}\left(\tau_{p} ; b_{p}\right) \text { in } \\
& L_{w_{p}}^{2}\left(a_{p}, b_{p}\right)=L_{w_{p}}^{2}\left(a_{p}, c_{p}\right) \oplus L_{w_{p}}^{2}\left(c_{p}, b_{p}\right), p=1,2, \cdots, N,
\end{aligned}
$$

$\tilde{T}_{0}^{\prime}\left(\tau_{p}\right)$ is densely-defined and closable in $L_{w_{p}}^{2}\left(a_{p}, b_{p}\right)$ and its closure is given by:

$$
\tilde{T}_{0}\left(\tau_{p}\right)=T_{0}\left(\tau_{p} ; a_{p}\right) \oplus T_{0}\left(\tau_{p} ; b_{p}\right), p=1,2, \cdots, N .
$$

Also,

$$
\begin{aligned}
& \operatorname{null}\left[\tilde{T}_{0}\left(\tau_{p}\right)-\lambda I\right] \\
& =\operatorname{null}\left[T_{0}\left(\tau_{p} ; a_{p}\right)-\lambda I\right]+\operatorname{null}\left[T_{0}\left(\tau_{p} ; b_{p}\right)-\lambda I\right], \\
& \operatorname{def}\left[\tilde{T}_{0}\left(\tau_{p}\right)-\lambda I\right] \\
& =\operatorname{def}\left[T_{0}\left(\tau_{p} ; a_{p}\right)-\lambda I\right]+\operatorname{def}\left[T_{0}\left(\tau_{p} ; b_{p}\right)-\lambda I\right]
\end{aligned}
$$


and $R\left[\tilde{T}_{0}\left(\tau_{p}\right)-\lambda I\right]$ is closed if and only if $R\left[T_{0}\left(\tau_{p} ; a_{p}\right)-\lambda I\right]$ and $R\left[T_{0}\left(\tau_{p} ; b_{p}\right)-\lambda I\right]$ are both closed. These results imply in particular that,

$$
\begin{aligned}
& \Pi\left[\tilde{T}_{0}\left(\tau_{p}\right)\right]=\Pi\left[T_{0}\left(\tau_{p} ; a_{p}\right)\right] \cap \Pi\left[T_{0}\left(\tau_{p} ; b_{p}\right)\right], \\
& p=1,2, \cdots, N .
\end{aligned}
$$

We refer to [11, Section 3.10.14], [16] and [18] for more details.

Remark 3.1: If $S_{p}^{a_{p}}$ is a regularly solvable extension of $T_{0}\left(\tau_{p} ; a_{p}\right)$ and $S_{p}^{p_{b_{p}}}$ is a regularly solvable extension of $T_{0}\left(\tau_{p} ; b_{p}\right)$, then $S=\oplus_{p=1}^{N}\left(S_{p}^{a_{p}} \oplus S_{p}^{b_{p}}\right)$ is a regularly solvable extension of $\tilde{T}_{0}(\tau)$. We refer to [11, Section 3.10.4], [16] and [18] for more details.

Next, we state the following results; the proof is similar to that in [10], [11, Section 3.10.4], [16] and [18].

Theorem 3.2: $\tilde{T}_{0}\left(\tau_{p}\right) \subset T_{0}\left(\tau_{p}\right)$,

$$
\begin{aligned}
& T\left(\tau_{p}\right) \subset T_{0}\left(\tau_{p} ; a_{p}\right) \oplus T_{0}\left(\tau_{p} ; b_{p}\right) \text { and } \\
& \quad \operatorname{dim}\left(D\left[T_{0}\left(\tau_{p}\right)\right] / D\left[\tilde{T}_{0}\left(\tau_{p}\right)\right]\right)=n, p=1,2, \cdots, N . \\
& \quad \text { If } \lambda \in \Pi\left[\tilde{T}_{0}\left(\tau_{p}\right)\right] \cap \Delta_{3}\left[T_{0}\left(\tau_{p}\right)-\lambda I\right], \text { then } \\
& \quad \operatorname{ind}\left[T_{0}\left(\tau_{p}\right)-\lambda I\right] \\
& \quad=n-\operatorname{def}\left[T_{0}\left(\tau_{p} ; a_{p}\right)-\lambda I\right]-\operatorname{def}\left[T_{0}\left(\tau_{p} ; b_{p}\right)-\lambda I\right],
\end{aligned}
$$

and in particular, if $\lambda \in \Pi\left[T_{0}\left(\tau_{p}\right)\right]$,

$$
\begin{aligned}
& \operatorname{def}\left[T_{0}\left(\tau_{p}\right)-\lambda I\right] \\
& =\operatorname{def}\left[T_{0}\left(\tau_{p} ; a_{p}\right)-\lambda I\right]+\operatorname{def}\left[T_{0}\left(\tau_{p} ; b_{p}\right)-\lambda I\right]-n .
\end{aligned}
$$

Remark 3.3: It can be shown that

$$
\begin{aligned}
& D\left[\tilde{T}_{0}\left(\tau_{p}\right)\right]: \\
& =\left\{u: u \in D\left[T_{0}\left(\tau_{p}\right)\right], u^{[r-1]}\left(c_{p}\right)=0, p=1,2, \cdots, N\right\} \\
& D\left[\tilde{T}_{0}\left(\tau_{p}^{+}\right)\right] \\
& :=\left\{v: v \in D\left[T_{0}\left(\tau_{p}^{+}\right)\right], v_{+}^{[r-1]}\left(c_{p}\right)=0, p=1,2, \cdots, N\right\} ;
\end{aligned}
$$

see [11, Section 3.10.4].

Let $H$ be the direct sum,

$$
H=\oplus_{p=1}^{N} H_{p}=\oplus_{p=1}^{N} L_{w_{p}}^{2}\left(a_{p}, b_{p}\right) .
$$

The elements of $H$ will be denoted by $\tilde{f}=\left\{f_{1}, f_{2}, \cdots, f_{N}\right\}$ with $f_{1} \in H_{1}$, $f_{2} \in H_{2}, \cdots, f_{N} \in H_{N}$.

\section{Remark 3.4:}

When $I_{i} \cap I_{j}=\varnothing, i \neq j ; i, j=1,2, \cdots, N$, the direct sum space $\oplus_{p=1}^{N} L_{w_{p}}^{2}\left(a_{p}, b_{p}\right)$ can be naturally identified with the space $L_{w}^{2}\left(\bigcup_{p=1}^{N} I_{p}\right)$, where $w_{p}=w$ on $I_{p}$, $p=1,2, \cdots, N$. This remark is of significance when $\left(\bigcup_{p=1}^{N} I_{p}\right)$, may be taken as a single interval, see [15] and [17].

We now establish by $[8,10,11,13,15]$ and $[18]$ and some further notations,

$$
\begin{aligned}
& D_{0}(\tau)=\oplus_{p=1}^{N} D_{0}\left(\tau_{p}\right), D(\tau)=\oplus_{p=1}^{N} D\left(\tau_{p}\right), \\
& D_{0}\left(\tau^{+}\right)=\oplus_{p=1}^{N} D_{0}\left(\tau_{p}^{+}\right), D\left(\tau^{+}\right)=\oplus_{p=1}^{N} D\left(\tau_{p}^{+}\right), \\
& T_{0}(\tau) f=\left\{T_{0}\left(\tau_{1}\right) f_{1}, T_{0}\left(\tau_{2}\right) f_{2}, \cdots, T_{0}\left(\tau_{N}\right) f_{N}\right\} ; \\
& \quad f_{1} \in D_{0}\left(\tau_{1}\right), f_{2} \in D_{0}\left(\tau_{2}\right), \cdots, f_{N} \in D_{0}\left(\tau_{N}\right), \\
& T_{0}\left(\tau^{+}\right) g=\left\{T_{0}\left(\tau_{1}^{+}\right) g_{1}, T_{0}\left(\tau_{2}^{+}\right) g_{2}, \cdots, T_{0}\left(\tau_{N}^{+}\right) g_{N}\right\} ; \\
& g_{1} \in D_{0}\left(\tau_{1}^{+}\right), g_{2} \in D_{0}\left(\tau_{2}^{+}\right), \cdots, g_{N} \in D_{0}\left(\tau_{N}^{+}\right) .
\end{aligned}
$$

Also,

$$
\begin{gathered}
T(\tau) f=\left\{T\left(\tau_{1}\right) f_{1}, T\left(\tau_{2}\right) f_{2}, \cdots, T\left(\tau_{N}\right) f_{N}\right\} \\
f_{1} \in D\left(\tau_{1}\right), f_{2} \in D\left(\tau_{2}\right), \cdots, f_{N} \in D\left(\tau_{N}\right), \\
T\left(\tau^{+}\right) g=\left\{T\left(\tau_{1}^{+}\right) g_{1}, T\left(\tau_{2}^{+}\right) g_{2}, \cdots, T\left(\tau_{N}^{+}\right) g_{N}\right\} \\
g_{1} \in D\left(\tau_{1}^{+}\right), g_{2} \in D\left(\tau_{2}^{+}\right), \cdots, g_{N} \in D\left(\tau_{N}^{+}\right) .
\end{gathered}
$$

We summarize a few additional properties of $T_{0}(\tau)$ in the form of a Lemma.

Lemma 3.5: We have,

i) $\left[T_{0}(\tau)\right]^{*}=\oplus_{p=1}^{N}\left[T_{0}\left(\tau_{p}\right)\right]^{*}=\oplus_{p=1}^{N}\left[T\left(\tau_{p}^{+}\right)\right]$,

$$
\left[T_{0}\left(\tau^{+}\right)\right]^{*}=\oplus_{p=1}^{N}\left[T_{0}\left(\tau_{p}^{+}\right)\right]^{*}=\oplus_{p=1}^{N}\left[T\left(\tau_{p}\right)\right] .
$$

In particular,

$$
\begin{aligned}
& D\left[T_{0}(\tau)\right]^{*}=D\left[T\left(\tau^{+}\right)\right]=\oplus_{p=1}^{N}\left[T\left(\tau_{p}^{+}\right)\right], \\
& D\left[T_{0}\left(\tau^{+}\right)\right]^{*}=D[T(\tau)]=\oplus_{p=1}^{N}\left[T\left(\tau_{p}\right)\right] .
\end{aligned}
$$

2) $\operatorname{null}\left[T_{0}(\tau)-\lambda I\right]=\sum_{p=1}^{N} \operatorname{null}\left[T_{0}\left(\tau_{p}\right)-\lambda I\right]$,

$$
\operatorname{null}\left[T_{0}\left(\tau^{+}\right)-\bar{\lambda} I\right]=\sum_{p=1}^{N} \text { null }\left[T_{0}\left(\tau_{p}^{+}\right)-\bar{\lambda} I\right] .
$$

3) The deficiency indices of $T_{0}(\tau)$ are given by:

$$
\operatorname{def}\left[T_{0}(\tau)-\lambda I\right]=\sum_{p=1}^{N} \operatorname{def}\left[T_{0}\left(\tau_{p}\right)-\lambda I\right]
$$

for all $\lambda \in \Pi\left[T_{0}\left(\tau_{p}\right)\right]$,

$$
\operatorname{def}\left[T_{0}\left(\tau^{+}\right)-\bar{\lambda} I\right]=\sum_{p=1}^{N} \operatorname{def}\left[T_{0}\left(\tau_{p}^{+}\right)-\bar{\lambda} I\right]
$$

for all $\lambda \in \Pi\left[T_{0}\left(\tau_{p}^{+}\right)\right]$.

Proof: Part (a) follows immediately from the definition of $T_{0}(\tau)$ and from the general definition of an adjoint operator. The other parts are either direct conse- 
quences of part (a) or follow immediately from the definitions.

Lemma 3.6: For $\lambda \in \Pi\left[T_{0}(\tau), T_{0}\left(\tau^{+}\right)\right]$, $\operatorname{def}\left[T_{0}(\tau)-\lambda I\right]+\operatorname{def}\left[T_{0}\left(\tau^{+}\right)-\bar{\lambda} I\right]$ is constant and

$$
0 \leq \operatorname{def}\left[T_{0}(\tau)-\lambda I\right]+\operatorname{def}\left[T_{0}\left(\tau^{+}\right)-\bar{\lambda} I\right] \leq 2 n N .
$$

In the problem with one singular end-point,

$$
n N \leq \operatorname{def}\left[T_{0}(\tau)-\lambda I\right]+\operatorname{def}\left[T_{0}\left(\tau^{+}\right)-\bar{\lambda} I\right] \leq 2 n N,
$$

for all $\lambda \in \Pi\left[T_{0}(\tau), T_{0}\left(\tau^{+}\right)\right]$.

In the regular problem,

$$
\operatorname{def}\left[T_{0}(\tau)-\lambda I\right]+\operatorname{def}\left[T_{0}\left(\tau^{+}\right)-\bar{\lambda} I\right]=2 n N,
$$

for all $\lambda \in \Pi\left[T_{0}(\tau), T_{0}\left(\tau^{+}\right)\right]$.

Proof: The proof is similar to that in [10, Lemma 2.4], [17] and [19] and therefore omitted.

Lemma 3.7: Let $T_{0}(\tau)=\oplus_{p=1}^{N} T_{0}\left(\tau_{p}\right)$ be a closed densely-defined operator on $H$. Then,

$$
\Pi\left[T_{0}(\tau)\right]=\bigcap_{p=1}^{N}\left[T_{0}\left(\tau_{p}\right)\right]
$$

Proof: The proof follows from Lemma 3.5 and since $R\left[T_{0}(\tau)-\lambda I\right]$ is closed if and only if $R\left[T_{0}\left(\tau_{p}\right)-\lambda I\right]$, $p=1,2, \cdots, N$ are closed.

Remark 3.8: If $S_{p}^{a_{p}}, p=1,2, \cdots, N$ is a regularly solvable extension of $T_{0}\left(\tau_{p} ; a_{p}\right)$ is a regularly solvable extension of $S_{p}^{b_{p}}$ then $T_{0}\left(\tau_{p} ; b_{p}\right)$ is regularly solvable extension of $S=\oplus_{p=1}^{N}\left(S_{p}^{a_{p}} \oplus S_{p}^{b_{p}}\right)$. We refer to $[10,11$, 16] and [19] for more details.

\section{The Product Operators}

The proof of general theorems will be based on the results in this section. We start by listing some properties and results of quasi-differential expressions $\tau_{1}, \tau_{2}, \cdots, \tau_{n}$. For proofs the reader is referred to $[3,8,10,17]$ and [19], and

$$
\begin{aligned}
& \left(\tau_{1}+\tau_{2}\right)^{+}=\tau_{1}^{+}+\tau_{2}^{+},\left(\tau_{1} \tau_{2}\right)^{+}=\tau_{2}^{+} \tau_{1}^{+}, \\
& (\lambda \tau)^{+}=\bar{\lambda} \tau^{+} \text {for } \lambda \text { a complex number. }
\end{aligned}
$$

A consequence of Properties (4.1) is that if $\tau^{+}=\tau$ then $(P(\tau))^{+}=P\left(\tau^{+}\right)$for $P$ any polynomial with complex coefficients. Also we note that the leading coefficients of a product are the product of the leading coefficients. Hence the product of regular differential expressions is regular.

Lemma 4.1: (cf. [19, Theorem 1]). Suppose $\tau_{j}$ is a regular differential expression on the interval $[a, b]$ and $\lambda \in \Pi\left[T_{0}\left(\tau_{1} \tau_{2} \cdots \tau_{n}\right), T_{0}\left(\tau_{1} \tau_{2} \cdots \tau_{n}\right)^{+}\right]$, then we have,
1) The product operator $\prod_{j=1}^{n} T_{0}\left(\tau_{j}\right)$ is closed, densely-defined, and

$$
\begin{aligned}
& \operatorname{def}\left[\prod_{j=1}^{n} T_{0}\left(\tau_{j}\right)-\lambda I\right]=\sum_{j=1}^{n} \operatorname{def}\left[T_{0}\left(\tau_{j}\right)-\lambda I\right], \\
& \operatorname{def}\left[\prod_{j=1}^{n} T_{0}\left(\tau_{j}^{+}\right)-\bar{\lambda} I\right]=\sum_{j=1}^{n} \operatorname{def}\left[T_{0}\left(\tau_{j}^{+}\right)-\bar{\lambda} I\right] . \\
& \text { 2) } T_{0}\left(\tau_{1} \tau_{2} \cdots \tau_{n}\right) \subseteq \prod_{j=1}^{n}\left[T_{0}\left(\tau_{j}\right)\right] \text { and } \\
& T_{0}\left(\tau_{1} \tau_{2} \cdots \tau_{n}\right)^{+} \subseteq \prod_{j=1}^{n}\left[T_{0}\left(\tau_{j}^{+}\right)\right] .
\end{aligned}
$$

Note in part (ii) that the containment may be proper, i.e., the operators $T_{0}\left(\tau_{1} \tau_{2} \cdots \tau_{n}\right)$ and $\prod_{j=1}^{n}\left[T_{0}\left(\tau_{j}\right)\right]$ are not equal in general.

Lemma 4.2: Let $\tau_{1}, \tau_{2}, \cdots, \tau_{n}$ be a regular differential expressions on $[a, b)$ and suppose that

$$
\begin{gathered}
\lambda \in \Pi\left[T_{0}\left(\tau_{1} \tau_{2} \cdots \tau_{n}\right), T_{0}\left(\tau_{1} \tau_{2} \cdots \tau_{n}\right)^{+}\right] . \text {Then } \\
{\left[T_{0}\left(\tau_{1} \tau_{2} \cdots \tau_{n}\right)\right]=\prod_{j=1}^{n}\left[T_{0}\left(\tau_{j}\right)\right]}
\end{gathered}
$$

if and only if the following partial separation conditions are satisfied:

$\left\{f \in L_{w}^{2}(a, b), f^{[s-1]} \in A C_{l o c}[a, b)\right.$, where $s$ is the order of product expression $\left(\tau_{1} \tau_{2} \cdots \tau_{n}\right)$ and $\left(\tau_{1} \tau_{2} \cdots \tau_{n}\right)^{+}$ $f \in L_{w}^{2}(a, b)$ together imply that:

$$
\left.\left(\prod_{j=1}^{k}\left(\tau_{j}^{+}\right)\right) f \in L_{w}^{2}(a, b), k=1, \cdots, n-1\right\} .
$$

Furthermore $T_{0}\left(\tau_{1} \tau_{2} \cdots \tau_{n}\right)=\prod_{j=1}^{n}\left[T_{0}\left(\tau_{j}\right)\right]$ and $T_{0}\left(\tau_{1} \tau_{2} \cdots \tau_{n}\right)^{+}=\prod_{j=1}^{n}\left[T_{0}\left(\tau_{j}^{+}\right)\right]$if and only if,

$$
\begin{gathered}
\operatorname{def}\left[T_{0}\left(\tau_{1} \tau_{2} \cdots \tau_{n}\right)-\lambda I\right]=\sum_{j=1}^{n} \operatorname{def}\left[T_{0}\left(\tau_{j}\right)-\lambda I\right], \\
\operatorname{def}\left[T_{0}\left(\tau_{1} \tau_{2} \cdots \tau_{n}\right)^{+}-\bar{\lambda} I\right]=\sum_{j=1}^{n} \operatorname{def}\left[T_{0}\left(\tau_{j}^{+}\right)-\bar{\lambda} I\right] .
\end{gathered}
$$

We will say that the product $\tau_{1}, \tau_{2}, \cdots, \tau_{n}$ is partially separated expressions in $L_{w}^{2}(a, b)$ whenever Property (4.3) holds.

Lemma 4.3: For

$\lambda \in \Pi\left[T_{0}\left(\tau_{1} \tau_{2} \cdots \tau_{n}\right), T_{0}\left(\tau_{1} \tau_{2} \cdots \tau_{n}\right)^{+}\right]$we have,

$$
\begin{aligned}
& \Pi\left[T_{0}\left(\tau_{1} \tau_{2} \cdots \tau_{n}\right), T_{0}\left(\tau_{1} \tau_{2} \cdots \tau_{n}\right)^{+}\right] \\
& =\Pi\left[\prod_{j=1}^{n}\left[T_{0}\left(\tau_{j}\right)\right], \prod_{j=1}^{n}\left[T_{0}\left(\tau_{j}^{+}\right)\right]\right] .
\end{aligned}
$$

Proof: Let $\lambda \in \Pi\left[T_{0}\left(\tau_{1} \tau_{2} \cdots \tau_{n}\right), T_{0}\left(\tau_{1} \tau_{2} \cdots \tau_{n}\right)^{+}\right]$, then from definition of the field of regularity we have $\lambda \in \Pi\left[T_{0}\left(\tau_{1} \tau_{2} \cdots \tau_{n}\right)\right]$ and $\lambda \in \Pi\left[T_{0}\left(\tau_{1} \tau_{2} \cdots \tau_{n}\right)^{+}\right]$, i.e., each of the operators $T_{0}\left(\tau_{1} \tau_{2} \cdots \tau_{n}\right)$ and $T_{0}\left(\tau_{1} \tau_{2} \cdots \tau_{n}\right)^{+}$ has closed range and densely-defined on $H$ with finite deficiency indices. Consequently by Lemma 4.2 each of the operators $\left[\prod_{j=1}^{n} T_{0}\left(\tau_{j}\right)-\lambda I\right]$ and 
$\left[\prod_{j=1}^{n} T_{0}\left(\tau_{j}^{+}\right)-\bar{\lambda} I\right]$ has closed range and their deficiency indices are finite, i.e.,

$\lambda \in \Pi\left[\prod_{j=1}^{n}\left[T_{0}\left(\tau_{j}\right)\right] \prod_{j=1}^{n}\left[T_{0}\left(\tau_{j}^{+}\right)\right]\right]$. The rest of the proof follows from definition and Lemma 4.2.

Corollary 4.4: Let $\tau_{j}$ is be a regular differential expression on $[a, b)$ for $j=1,2, \cdots, n$ If all solutions of the differential equation $\left(\tau_{j}-\lambda I\right) u=0$ and

$\left(\tau_{j}^{+}-\bar{\lambda} I\right) \nu=0$ on $[a, b)$ are in $L_{w}^{2}(a, b)$ for

$j=1,2, \cdots, n$ and $\lambda \in \mathbb{C}$; then all solutions of

$\left[\prod_{j=1}^{n} \tau_{j}-\lambda I\right] u=0$ and $\left(\prod_{j=1}^{n} \tau_{j}^{+}-\bar{\lambda} I\right) v=0$ on

$[a, b)$ are in $L_{w}^{2}(a, b)$ for all $\lambda \in \mathbb{C}$.

Proof: Let $n=n_{j}=$ order of $\tau_{j}=$ order of $\tau_{j}^{+}$for $j=1,2, \cdots, n$. Then by Lemma 2.5 , we have

$$
\operatorname{def}\left[T_{0}(\tau)-\lambda I\right]=\operatorname{def}\left[T_{0}\left(\tau^{+}\right)-\bar{\lambda} I\right]=n
$$

for all $\lambda \in \Pi\left[T_{0}(\tau), T_{0}\left(\tau^{+}\right)\right]$.

Hence, by Lemma 4.1, we have,

$$
\begin{aligned}
& \operatorname{def}\left[T_{0}\left(\tau_{1} \tau_{2} \cdots \tau_{n}\right)^{+}-\lambda I\right] \\
& =\operatorname{def}\left[\prod_{j=1}^{n} T_{0}\left(\tau_{j}^{+}\right)-\bar{\lambda} I\right]=\sum_{j=1}^{n} n_{j}=n^{2} \\
& =\operatorname{order} \text { of }\left(\tau_{1} \tau_{2} \cdots \tau_{n}\right)=\operatorname{order} \text { of }\left(\tau_{1} \tau_{2} \cdots \tau_{n}\right)^{+} .
\end{aligned}
$$

Thus $\operatorname{def}\left[T_{0}\left(\tau_{n}^{+} \cdots \tau_{2}^{+} \tau_{1}^{+}\right)-\lambda I\right]=$ order of $\left(\tau_{1} \tau_{2} \cdots \tau_{n}\right)^{+}$ and consequently all solutions of the equations

$\left[\prod_{j=1}^{n} \tau_{j}-\lambda I\right] u=0$ and $\left(\prod_{j=1}^{n} \tau_{j}^{+}-\bar{\lambda} I\right) \nu=0$ are in $L_{w}^{2}(a, b)$. Repeating this argument with $\tau_{j}^{+}$replaced by $\tau_{j}$, we conclude that all solutions of

$\left(\prod_{j=1}^{n} \tau_{j}^{+}-\bar{\lambda} I\right) v=0$ are in $L_{w}^{2}(a, b)$.

The special case of Corollary 4.4 when $\tau_{j}=\tau$ for $j=1,2, \cdots, n$ and $\tau$ is symmetric was established in [9]. In this case it is easy to see that the converse also holds. If all solutions of $\left(\tau^{n}-\lambda I\right) u=0$ are in $L_{w}^{2}(a, b)$, then all solutions of $(\tau-\lambda I) u=0$ must be in $L_{w}^{2}(a, b)$. In general, if all solutions of $\left[\left(\tau_{1} \tau_{2} \cdots \tau_{n}\right)-\lambda I\right] u=0$ are in $L_{w}^{2}(a, b)$, then all solutions of $\left(\tau_{n}-\lambda I\right) u=0$ are in $L_{w}^{2}(a, b)$ since these are also solutions of $\left[\left(\tau_{1} \tau_{2} \cdots \tau_{n}\right)-\lambda I\right] u=0$. If all solutions of the adjoint equation $\left[\left(\tau_{1} \tau_{2} \cdots \tau_{n}\right)^{+}-\bar{\lambda} I\right] v=0$ are also in $L_{w}^{2}(a, b)$, then it follows similarly that all solutions of $\left(\tau_{j}^{+}-\bar{\lambda} I\right) v=0$ are in $L_{w}^{2}(a, b)$.

Let $\varphi_{k}(t, \lambda), k=1,2, \cdots, n^{2}$ be the solutions of the homogeneous equation

$$
\left[\prod_{j=1}^{n} \tau_{j}-\lambda I\right] u=0(\lambda \in \mathbb{C})
$$

satisfying $\varphi_{j}^{[k-1]}\left(t_{0}, \lambda\right)=\delta_{k, r+1}$ for all $t_{0} \in[a, b)$ $\left(j, k=1,2, \cdots, n^{2}, r=0,1, \cdots, n^{2}-1\right)$ for fixed $t_{0}, a<t_{0}<b$. Then $\varphi_{j}^{[r]}(t, \lambda)$ is continuous in $(t, \lambda)$ for $a<t<b,|\lambda|<\infty$, and for fixed $t$ it is entire in $\lambda$. Let $\varphi_{k}^{+}(t, \lambda), k=1,2, \cdots, n^{2}$ denote the solutions of the adjoint homogeneous equation

$$
\left(\prod_{j=1}^{n} \tau_{j}^{+}-\bar{\lambda} I\right) \nu=0(\lambda \in \mathbb{C})
$$

satisfying $\left(\varphi_{k}^{+}\right)^{[r]}\left(t_{0}, \lambda\right)=(-1)^{k+r} \delta_{k, n^{2}-r}$ for all $t_{0} \in[a, b) \quad\left(j, k=1,2, \cdots, n^{2}, r=0,1, \cdots, n^{2}-1\right)$.

Suppose $a<c<b$ By [8], a solution of the product equation

$$
\left[\prod_{j=1}^{n} \tau_{j}-\lambda I\right] u=w f \quad(\lambda \in \mathbb{C}), f \in L_{w}^{1}(a, b)
$$

satisfying $u^{[r]}(c)=0, r=0,1, \cdots, n^{2}-1$ is given by,

$$
\varphi(t)=\left(\frac{1}{i^{n}}\right) \sum_{j, k=1}^{n^{2}} \xi^{j k} \varphi_{j}(t, \lambda) \int_{a}^{t} \overline{\varphi_{k}^{+}(t, \lambda)} f(s) w(s) \mathrm{d} s,
$$

where $\varphi_{k}^{+}(t, \lambda)$ stands for the complex conjugate of $\varphi_{k}(t, \lambda)$ and for each $j, k, \xi^{j k}$ is constant which is independent of $t, \lambda$ (but does depend in general on $t_{0}$ ).

The next lemma is a form of the variation of parameters formula for a general quasi-differential equation is given by the following Lemma.

Lemma 4.5: Suppose $f \in L_{w}^{1}(a, b)$ locally integrable function and $\varphi(t, \lambda)$ is the solution of the Equation (4.7) satisfying:

$$
\varphi^{[r]}\left(t_{0}, \lambda\right)=\alpha_{r+1} \text { for } r=0,1, \cdots, n^{2}-1, t_{0} \in[a, b) \text { is }
$$

given by

$$
\begin{aligned}
& \varphi(t, \lambda)=\sum_{j=1}^{n^{2}} \alpha_{j}(\lambda) \varphi_{j}\left(t, \lambda_{0}\right) \\
& +\frac{1}{i^{n^{2}}}\left(\lambda-\lambda_{0}\right) \sum_{j, k=1}^{n^{2}} \xi^{j k} \varphi_{j}\left(t, \lambda_{0}\right) \int_{a}^{t} \overline{\varphi_{k}^{+}\left(t, \lambda_{0}\right)} f(s) w(s) \mathrm{d} s
\end{aligned}
$$

for some constants $\alpha_{1}(\lambda), \alpha_{2}(\lambda), \cdots, \alpha_{n_{2}^{2}}(\lambda) \in \mathbb{C}$, where $\varphi_{j}\left(t, \lambda_{0}\right)$ and $\varphi_{k}^{+}\left(t, \lambda_{0}\right), j, k=1,2, \cdots, n^{n_{2}^{2}}$ are solutions of the Equations (4.5) and (4.6) respectively, $\xi^{j k}$ is a constant which is independent of $t$.

Proof: The proof is similar to that in $[2,9,13,15,17]$. Lemma 4.5 contains the following lemma as a special case.

Lemma 4.6: Suppose $f \in L_{w}^{1}(a, b)$ locally integrable function and $\varphi(t, \lambda)$ is the solution of Equation (4.7) satisfying:

$$
\varphi^{[r]}\left(t_{0}, \lambda\right)=\alpha_{r+1} \text { for } r=0,1, \cdots, n^{2}-1, t_{0} \in[a, b) .
$$

Then

$$
\begin{aligned}
& \varphi^{[r]}(t, \lambda)=\sum_{j=1}^{n^{2}} \alpha_{j}(\lambda) \varphi_{j}^{[r]}\left(t, \lambda_{0}\right) \\
& +\frac{1}{i^{n^{2}}}\left(\lambda-\lambda_{0}\right) \sum_{j, k=1}^{n^{2}} \xi^{j k} \varphi_{j}^{[r]}\left(t, \lambda_{0}\right) \\
& \times \int_{a}^{t} \overline{\varphi_{k}^{+}\left(t, \lambda_{0}\right)} f(s) w(s) \mathrm{d} s
\end{aligned}
$$


for $r=1, \cdots, n^{2}-1$. We refer to [20] for more details.

Lemma 4.7: Suppose that for some $\lambda_{0} \in \mathbb{C}$ all solutions of the equations

$$
\left[\prod_{j=1}^{n} \tau_{j}-\lambda_{0} I\right] \varphi=0,\left(\prod_{j=1}^{n} \tau_{j}^{+}-\overline{\lambda_{0}} I\right) \varphi^{+}=0
$$

are in $L_{w}^{2}(a, b)$. Then all solutions of the equations in (4.10) are in $L_{w}^{2}(a, b)$ for every complex number $\lambda \in \mathbb{C}$.

Proof: The proof is similar to that in [17, Theorem 5.3].

Lemma 4.8: Suppose that for some complex number $\lambda_{0} \in \mathbb{C}$ all solutions of the equations in (4.10) are in $L_{w}^{2}(a, b)$. Suppose $f \in L_{w}^{2}(a, b)$. Then all solutions of the equation (4.7) are in $L_{w}^{2}(a, b)$ for all $\lambda \in \mathbb{C}$.

Proof: The proof is similar to that in [19, Lemma 3.8].

Remark 4.9: Lemma 4.8 also holds if the function $f$ is bounded on $[a, b)$.

Lemma 4.10: Let $f \in L_{w}^{2}(a, b)$. Suppose for some $\lambda_{0} \in \mathbb{C}$ that:

1) All solutions of $\left(\prod_{j=1}^{n} \tau_{j}^{+}-\bar{\lambda} I\right) \varphi^{+}=0$ are in $L_{w}^{2}(a, b)$.

2) $\varphi_{j}^{[r]}\left(t, \lambda_{0}\right), j=1, \cdots, n^{2}$ are bounded on $[a, b)$ for some $r=1, \cdots, n^{2}-1$.

Then $\varphi^{[r]}(t, \lambda) \in L_{w}^{2}(a, b)$ for any solution $\varphi(t, \lambda)$ of the equation $\left[\prod_{j=1}^{n} \tau_{j}-\lambda I\right] \varphi=w f$ for all $\lambda \in \mathbb{C}$.

Lemma 4.11: Suppose that for some complex $\lambda_{0} \in \mathbb{C}$ all solutions of the equation $\left(\prod_{j=1}^{n} \tau_{j}^{+}-\bar{\lambda} I\right) v=0$ are in $L_{w}^{2}(a, c)$, where $a<c<b$. Suppose $f \in L_{w}^{2}(a, b)$, then $\int_{a}^{t} \overline{\varphi_{k}^{+}\left(t, \lambda_{0}\right)} f(s) w(s) \mathrm{d} s, \quad j=1,2, \cdots, n^{2}$ is continuous in $(t, \lambda)$ for $a<t<b$ and for all $\lambda \in \mathbb{C}$.

Proof: It follows from Lemma 4.8; see [18, Lemma 3.6].

Lemma 4.12: The point spectra $\sigma_{p}\left[\prod_{j=1}^{n} T_{0}\left(\tau_{j}\right)\right]$ and $\sigma_{p}\left[\prod_{j=1}^{n} T_{0}\left(\tau_{j}^{+}\right)\right]$of the product operators $\prod_{j=1}^{n} T_{0}\left(\tau_{j}\right)$ and $\prod_{j=1}^{n} T_{0}\left(\tau_{j}^{+}\right)$are empty.

Proof: See [17, Theorem 4.6].

Lemma 4.13: If $I=[a, b]$, with $-\infty<a<b<\infty$ then for any $\lambda \in \mathbb{C}$, the operator $\prod_{j=1}^{n} T_{0}\left(\tau_{j}\right)$ has closed range, zero nullity and deficiency $n^{2}$. Hence,

$$
\sigma_{e k}\left[\prod_{j=1}^{n} T_{0}\left(\tau_{j}\right)\right]=\left\{\begin{array}{lc}
\varnothing & (k=1,2,3) \\
\mathbb{C} & (k=4,5)
\end{array}\right.
$$

Proof: The proof is similar to that in [11, Lemma IX.9.11]; see [17, Lemma 4.9].

\section{The Product Operators in Direct Sum Spaces}

Next, we consider our interval is $I=[a, b]$ and denote by $T_{0}\left(\tau_{1} \tau_{2} \cdots \tau_{n}\right)$ and $T\left(\tau_{1} \tau_{2} \cdots \tau_{n}\right)$ the minimal and maximal operators. We see from (3.15) and Lemma 4.2 that $T_{0}\left(\tau_{1} \tau_{2} \cdots \tau_{n}\right) \subset T\left(\tau_{1} \tau_{2} \cdots \tau_{n}\right) \subset\left[T_{0}\left(\tau_{1} \tau_{2} \cdots \tau_{n}\right)^{+}\right]^{*}$ and hence $T_{0}\left(\tau_{1} \tau_{2} \cdots \tau_{n}\right)$ and $T_{0}\left(\tau_{1} \tau_{2} \cdots \tau_{n}\right)^{+}$form an adjoint pair of closed densely defined operators in $L_{w}^{2}(a, b)$. From Lemmas 3.5 and 4.1 we have the following:

Lemma 5.1: For

$\lambda \in \Pi\left[\prod_{j=1}^{n}\left[T_{0}\left(\tau_{j}\right)\right], \prod_{j=1}^{n}\left[T_{0}\left(\tau_{j}^{+}\right)\right]\right]$we have:

1) $\left[\prod_{j=1}^{n} T_{0}^{*}\left(\tau_{j}\right)\right]=\oplus_{p=1}^{N}\left[\prod_{j=1}^{n} T_{0}^{*}\left(\tau_{j p}\right)\right]$ $=\oplus_{p=1}^{N}\left[\prod_{j=1}^{n} T\left(\tau_{j p}\right)\right]$,

$\left[\prod_{j=1}^{n} T_{0}^{*}\left(\tau_{j}^{+}\right)\right]=\oplus_{p=1}^{N}\left[\prod_{j=1}^{n} T_{0}^{*}\left(\tau_{j p}^{+}\right)\right]$

$=\oplus_{p=1}^{N}\left[\prod_{j=1}^{n} T\left(\tau_{j p}\right)\right]$.

2) $\operatorname{null}\left[\prod_{j=1}^{n} T_{0}\left(\tau_{j}\right)-\lambda I\right]$

$=\sum_{p=1}^{N} \operatorname{null}\left[\prod_{j=1}^{n} T_{0}\left(\tau_{j p}\right)-\lambda I\right]$

$=\sum_{p=1}^{N}\left(\sum_{j=1}^{n} n u l l\left[\prod_{j=1}^{n} T_{0}\left(\tau_{j r}\right)-\lambda I\right]\right)$

$\operatorname{null}\left[\prod_{j=1}^{n} T_{0}\left(\tau_{j}^{+}\right)-\bar{\lambda} I\right]$

$=\sum_{p=1}^{N} n u l l\left[\prod_{j=1}^{n} T_{0}\left(\tau_{j p}^{+}\right)-\bar{\lambda} I\right]$

$=\sum_{p=1}^{N}\left(\sum_{j=1}^{n} n u l l\left[\prod_{j=1}^{n} T_{0}\left(\tau_{j p}^{+}\right)-\bar{\lambda} I\right]\right)$

3) The deficiency indices of $\prod_{j=1}^{n} T_{0}\left(\tau_{j}\right)$ and $\prod_{j=1}^{n} T_{0}\left(\tau_{j}^{+}\right)$are given by:

$\operatorname{def}\left[\prod_{j=1}^{n} T_{0}\left(\tau_{j}\right)-\lambda I\right]=\sum_{p=1}^{N} \operatorname{def}\left[\prod_{j=1}^{n} T_{0}\left(\tau_{j p}\right)-\lambda I\right]$

$=\sum_{p=1}^{N}\left(\sum_{j=1}^{n} \operatorname{def}\left[\prod_{j=1}^{n} T_{0}\left(\tau_{j p}\right)-\lambda I\right]\right)$,

$\operatorname{def}\left[\prod_{j=1}^{n} T_{0}\left(\tau_{j}^{+}\right)-\bar{\lambda} I\right]=\sum_{p=1}^{N} \operatorname{def}\left[\prod_{j=1}^{n} T_{0}\left(\tau_{j p}^{+}\right)-\bar{\lambda} I\right]$

$=\sum_{p=1}^{N}\left(\sum_{j=1}^{n} \operatorname{def}\left[\prod_{j=1}^{n} T_{0}\left(\tau_{j p}^{+}\right)-\bar{\lambda} I\right]\right)$.

Lemma 5.2: For

$$
\begin{gathered}
\lambda \in \Pi\left[\prod_{j=1}^{n}\left[T_{0}\left(\tau_{j}\right)\right], \prod_{j=1}^{n}\left[T_{0}\left(\tau_{j}^{+}\right)\right]\right], \\
\operatorname{def}\left[\prod_{j=1}^{n}\left[T_{0}\left(\tau_{j}\right)\right]-\lambda I\right]+\operatorname{def}\left[\prod_{j=1}^{n}\left[T_{0}\left(\tau_{j}^{+}\right)\right]-\bar{\lambda} I\right]
\end{gathered}
$$

is constant and

$$
\begin{aligned}
& 0 \leq \operatorname{def}\left[\prod_{j=1}^{n}\left[T_{0}\left(\tau_{j}\right)\right]-\lambda I\right] \\
& +\operatorname{def}\left[\prod_{j=1}^{n}\left[T_{0}\left(\tau_{j}^{+}\right)\right]-\bar{\lambda} I\right] \leq 2 n^{2} N .
\end{aligned}
$$

In the problem with one singular end-point, 


$$
\begin{aligned}
& n^{2} N \leq \operatorname{def}\left[\prod_{j=1}^{n}\left[T_{0}\left(\tau_{j}\right)\right]-\lambda I\right] \\
& +\operatorname{def}\left[\prod_{j=1}^{n}\left[T_{0}\left(\tau_{j}^{+}\right)\right]-\bar{\lambda} I\right] \leq 2 n^{2} N .
\end{aligned}
$$

for all $\lambda \in \Pi\left[\prod_{j=1}^{n}\left[T_{0}\left(\tau_{j}\right)\right], \prod_{j=1}^{n}\left[T_{0}\left(\tau_{j}^{+}\right)\right]\right]$.

In the regular problem,

$$
\begin{aligned}
& \operatorname{def}\left[\prod_{j=1}^{n}\left[T_{0}\left(\tau_{j}\right)\right]-\lambda I\right] \\
& +\operatorname{def}\left[\prod_{j=1}^{n}\left[T_{0}\left(\tau_{j}^{+}\right)\right]-\bar{\lambda} I\right]=2 n^{2} N,
\end{aligned}
$$

for all $\lambda \in \Pi\left[\prod_{j=1}^{n}\left[T_{0}\left(\tau_{j}\right)\right], \prod_{j=1}^{n}\left[T_{0}\left(\tau_{j}^{+}\right)\right]\right]$.

Proof: The proof is similar to that in [10, Lemma 2.4], [17] and [19] and therefore omitted.

For $\lambda \in \Pi\left[\prod_{j=1}^{n}\left[T_{0}\left(\tau_{j}\right)\right], \prod_{j=1}^{n}\left[T_{0}\left(\tau_{j}^{+}\right)\right]\right]$, we define $r, s$ and $m$ as follows:

$$
\begin{aligned}
r & =r(\lambda):=\operatorname{def}\left[\prod_{j=1}^{n}\left[T_{0}\left(\tau_{j}\right)\right]-\lambda I\right] \\
& =\sum_{p=1}^{N} \operatorname{def}\left[\prod_{j=1}^{n} T\left(\tau_{j p}\right)-\lambda I\right]=\sum_{p=1}^{N} r_{p}, \\
s & =s(\lambda):=\operatorname{def}\left[\prod_{j=1}^{n}\left[T_{0}\left(\tau_{j}^{+}\right)\right]-\bar{\lambda} I\right] \\
& =\sum_{p=1}^{N} \operatorname{def}\left[\prod_{j=1}^{n} T\left(\tau_{j p}^{+}\right)-\bar{\lambda} I\right]=\sum_{p=1}^{N} s_{p},
\end{aligned}
$$

and

$$
m=r+s=\sum_{p=1}^{N}\left(r_{p}+s_{p}\right)
$$

Also,

$$
0 \leq m \leq 2 n^{2} N
$$

For $\Pi\left[\prod_{j=1}^{n}\left[T_{0}\left(\tau_{j}\right)\right], \prod_{j=1}^{n}\left[T_{0}\left(\tau_{j}^{+}\right)\right]\right] \neq \varnothing$ the operators which are regularly solvable with respect to $\prod_{j=1}^{n}\left[T_{0}\left(\tau_{j}\right)\right]$ and $\prod_{j=1}^{n}\left[T_{0}\left(\tau_{j}^{+}\right)\right]$are characterized by the following theorem which proved for a general quasi-differential operator in [11, Theorem 10.15].

Theorem 5.3: For $\lambda \in \Pi\left[\prod_{j=1}^{n}\left[T_{0}\left(\tau_{j}\right)\right], \prod_{j=1}^{n}\left[T_{0}\left(\tau_{j}^{+}\right)\right]\right]$. Let $r, s$ and $m$ be defined by (5.1) and (5.2), and let $\psi_{j}(j=1,2, \cdots, r), \Phi_{k}(k=r+1, \cdots, m)$ be arbitrary functions satisfying:

1) $\psi_{j}(j=1,2, \cdots, r) \subset D\left[\prod_{j=1}^{n} T\left(\tau_{j}\right)\right]$ are linearly independent modulo $D\left[\prod_{j=1}^{n} T\left(\tau_{j}\right)\right]$ and $\Phi_{k}(k=r+1, \cdots, m) \subset D\left[\prod_{j=1}^{n} T\left(\tau_{j}^{+}\right)\right]$are linearly independent modulo $D\left[\prod_{j=1}^{n} T\left(\tau_{j}^{+}\right)\right]$.

2) $\left[\psi_{j}, \Phi_{k}\right](b)-\left[\psi_{j}, \Phi_{k}\right](a)=0$, $(j=1,2, \cdots, r ; k=r+1, \cdots, m)$.
Then the set

$$
\begin{aligned}
& \left\{u: u \in D\left[\prod_{j=1}^{n} T\left(\tau_{j}\right)\right]\right. \\
& \left.\quad\left[u, \Phi_{k}\right](b)-\left[u, \Phi_{k}\right](a)=0, \quad k=r+1, \cdots, m\right\},
\end{aligned}
$$

is the domain of an operator $S$ which is regularly solvable with respect to $\left[\prod_{j=1}^{n} T_{0}\left(\tau_{j}\right)\right]$ and $\left[\prod_{j=1}^{n} T_{0}\left(\tau_{j}^{+}\right)\right]$and the set

$$
\begin{aligned}
& \left\{v: v \in D\left[\prod_{j=1}^{n} T\left(\tau_{j}^{+}\right)\right],\right. \\
& \left.\quad\left[\psi_{j}, v\right](b)-\left[\psi_{j}, v\right](a)=0, \quad j=1,2, \cdots, r\right\}
\end{aligned}
$$

is the domain of the operator $S^{*}$; moreover $\lambda \in \Delta_{4}(S)$.

Conversely, if $S$ is regularly solvable with respect to $\left[\prod_{j=1}^{n} T_{0}\left(\tau_{j}\right)\right]$ and $\left[\prod_{j=1}^{n} T_{0}\left(\tau_{j}^{+}\right)\right]$and $\lambda \in \Pi\left[\prod_{j=1}^{n}\left[T_{0}\left(\tau_{j}\right)\right], \prod_{j=1}^{n}\left[T_{0}\left(\tau_{j}^{+}\right)\right]\right] \cap \Delta_{4}(S)$, then

with $r$ and $s$ defined by (5.1) and (5.2) there exist functions $\psi_{j}(j=1,2, \cdots, r), \Phi_{k}(k=r+1, \cdots, m)$ which satisfy (1) and (2) and are such that (5.4) and (5.5) are the domains of $S$ and $S^{*}$ respectively.

$S$ is self-adjoint if, and only if,

$\prod_{j=1}^{n}\left(\tau_{j}\right)=\prod_{j=1}^{n}\left(\tau_{j}^{+}\right), r=s$ and

$\Phi_{k}=\psi_{k-r}(k=r+1, \cdots, m) ; \quad S$ is $J$-self-adjoint if $\prod_{j=1}^{n}\left(\tau_{j}\right)=J \prod_{j=1}^{n}\left(\tau_{j}\right) J$ ( $J$ is a complex conjugate), $r=s$ and $\Phi_{k}=\bar{\psi}_{k-r}(k=r+1, \cdots, m)$.

Proof: The proof is entirely similar to that of $[11,13$, 16,18] and [19] and therefore omitted.

\section{The Case of One Singular End-Point}

We see from (3.15) and Lemma 4.2 that $\prod_{j=1}^{n}\left[T_{0}\left(\tau_{j}\right)\right] \subset \prod_{j=1}^{n}\left[T\left(\tau_{j}\right)\right]=\prod_{j=1}^{n}\left[T_{0}\left(\tau_{j}^{+}\right)\right]^{*}$ and hence $\prod_{j=1}^{n}\left[T_{0}\left(\tau_{j}\right)\right]$ and $\prod_{j=1}^{n}\left[T_{0}\left(\tau_{j}^{+}\right)\right]$form an adjoint pair of closed, closed-densely operators in $L_{w}^{2}(a, b)$. By Lemmas 3.1, 3.2, [10, Lemma 2.4] and [17, Lemma 3.1],

$$
\operatorname{def}\left[\prod_{j=1}^{n}\left[T_{0}\left(\tau_{j}\right)\right]-\lambda I\right]+\operatorname{def}\left[\prod_{j=1}^{n}\left[T_{0}\left(\tau_{j}^{+}\right)\right]-\bar{\lambda} I\right]
$$

is constant on the joint field of regularity $\Pi\left[\prod_{j=1}^{n}\left[T_{0}\left(\tau_{j}\right)\right], \prod_{j=1}^{n}\left[T_{0}\left(\tau_{j}^{+}\right)\right]\right]$and we have that,

$$
\begin{aligned}
& n^{2} N \leq \operatorname{def}\left[\prod_{j=1}^{n}\left[T_{0}\left(\tau_{j}\right)\right]-\lambda I\right] \\
& +\operatorname{def}\left[\prod_{j=1}^{n}\left[T_{0}\left(\tau_{j}^{+}\right)\right]-\bar{\lambda} I\right] \leq 2 n^{2} N .
\end{aligned}
$$


for all $\lambda \in \Pi\left[\prod_{j=1}^{n}\left[T_{0}\left(\tau_{j}\right)\right], \prod_{j=1}^{n}\left[T_{0}\left(\tau_{j}^{+}\right)\right]\right]$.

We shall use the notation

$$
\begin{aligned}
& {[u, v](b)=\lim _{x \rightarrow b^{-}}[u, v](x)} \\
& u \in D\left[\prod_{j=1}^{n} T\left(\tau_{j}\right)\right], v \in D\left[\prod_{j=1}^{n} T\left(\tau_{j}^{+}\right)\right],
\end{aligned}
$$

if $b$ is a singular end-point of $[a, b)$, and similarly for $[u, v](a)$ if $a$ is singular.

Note that, it follows from (3.6) that these limits exist for $u \in D\left[\prod_{j=1}^{n} T\left(\tau_{j}\right)\right]$ and $v \in D\left[\prod_{j=1}^{n} T\left(\tau_{j}^{+}\right)\right]$

since then $v\left(\prod_{j=1}^{n}\left(\tau_{j}\right)\right)[\bar{u}]$ and $u\left(\overline{\prod_{j=1}^{n}\left(\tau_{j}^{+}\right)[v]}\right)$ are both integrable by Cauchy-Schwartz inequality.

We shall now investigate in the case of one singular end-point that the resolvent of all well-posed extensions of the minimal operator $\prod_{j=1}^{n}\left[T_{0}\left(\tau_{j}\right)\right]$, and we show that in the maximal case, i.e., when

$$
\begin{aligned}
& \operatorname{def}\left[\prod_{j=1}^{n}\left[T_{0}\left(\tau_{j}\right)\right]-\lambda I\right] \\
& =\operatorname{def}\left[\prod_{j=1}^{n}\left[T_{0}\left(\tau_{j}^{+}\right)\right]-\bar{\lambda} I\right]=n^{2} N
\end{aligned}
$$

for all $\lambda \in \Pi\left[\prod_{j=1}^{n}\left[T_{0}\left(\tau_{j}\right)\right], \prod_{j=1}^{n}\left[T_{0}\left(\tau_{j}^{+}\right)\right]\right]$, these resolvent are integral operators, in fact they are Hilbert-Schmidt integral operators by considering that the function $f$ be in $L_{w}^{2}(a, b)$, i.e., is quadratically integrable over the interval $[a, b)$.

Theorem 6.1: Suppose for an operator $\prod_{j=1}^{n}\left[T_{0}\left(\tau_{j}\right)\right]$ with one singular end-point that,

$$
\begin{aligned}
& \operatorname{def}\left[\prod_{j=1}^{n}\left[T_{0}\left(\tau_{j}\right)\right]-\lambda I\right] \\
& =\operatorname{def}\left[\prod_{j=1}^{n}\left[T_{0}\left(\tau_{j}^{+}\right)\right]-\bar{\lambda} I\right]=n^{2} N
\end{aligned}
$$

for all $\lambda \in \Pi\left[\prod_{j=1}^{n}\left[T_{0}\left(\tau_{j}\right)\right], \prod_{j=1}^{n}\left[T_{0}\left(\tau_{j}^{+}\right)\right]\right]$and let $S$ be an arbitrary closed operator which is a well-posed extension of the minimal operator $\prod_{j=1}^{n}\left[T_{0}\left(\tau_{j}\right)\right]$ and $\lambda \in \rho(S)$, then the resolvents $R_{\lambda}$ and $R_{\lambda}^{*}$ of $S$ and $S^{*}$ respectively are Hilbert-Schmidt integral operators whose kernels are continuous functions on $[a, b) \times[a, b)$ and satisfy:

$$
\begin{aligned}
& K(t, s, \lambda)=\overline{K^{+}(s, t, \lambda)} \\
& \text { and } \int_{a}^{b}|K(t, s, \lambda)|^{2} w(s) w(t) \mathrm{d} s \mathrm{~d} t<\infty
\end{aligned}
$$

Remark An example of a closed operator which is a well-posed with respect to a compatible adjoint pair is given by the Visik extension (see [6,7,11, Theorem III.3.3], [19] and [20, Theorem 1]). Note that if $S$ is well-posed, then $\prod_{j=1}^{n}\left[T_{0}\left(\tau_{j}\right)\right]$ and $\prod_{j=1}^{n}\left[T_{0}\left(\tau_{j}^{+}\right)\right]$ are compatible adjoint pair and $S$ is regularly solvable with respect to $\prod_{j=1}^{n}\left[T_{0}\left(\tau_{j}\right)\right]$ and $\prod_{j=1}^{n}\left[T_{0}\left(\tau_{j}^{+}\right)\right]$.

Proof: Let

$$
\begin{aligned}
& \operatorname{def}\left[\prod_{j=1}^{n}\left[T_{0}\left(\tau_{j}\right)\right]-\lambda I\right] \\
& =\operatorname{def}\left[\prod_{j=1}^{n}\left[T_{0}\left(\tau_{j}^{+}\right)\right]-\bar{\lambda} I\right]=n^{2} N
\end{aligned}
$$

for all $\lambda \in \Pi\left[\prod_{j=1}^{n}\left[T_{0}\left(\tau_{j}\right)\right], \prod_{j=1}^{n}\left[T_{0}\left(\tau_{j}^{+}\right)\right]\right]$, then we choose a fundamental system of solutions

$\left\{\varphi_{1}(t, \lambda), \varphi_{2}(t, \lambda), \cdots, \varphi_{n^{2} N}(t, \lambda)\right\}$,

$\left\{\varphi_{1}^{+}(t, \lambda), \varphi_{2}^{+}(t, \lambda), \cdots, \varphi_{n^{2} N}^{+}(t, \lambda)\right\}$ of the equations,

$$
\begin{aligned}
& {\left[\prod_{j=1}^{n}\left[T_{0}\left(\tau_{j}\right)\right]-\lambda I\right] \varphi_{j}=0,} \\
& {\left[\prod_{j=1}^{n}\left[T_{0}\left(\tau_{j}^{+}\right)\right]-\bar{\lambda} I\right] \varphi_{k}^{+}=0} \\
& \left(j, k=1, \cdots, n^{2} N\right) \text { on }[a, b),
\end{aligned}
$$

so that $\left\{\varphi_{1}(t, \lambda), \varphi_{2}(t, \lambda), \cdots, \varphi_{n^{2} N}(t, \lambda)\right\}$, $\left\{\varphi_{1}^{+}(t, \lambda), \varphi_{2}^{+}(t, \lambda), \cdots, \varphi_{n^{2} N}^{+}(t, \lambda)\right\}$ belong to $L_{w}^{2}(a, b)$ i.e., they are quadratically integrable in the interval $[a, b)$. Let $R_{\lambda}=(S-\lambda I)^{-1}$ be the resolvent of any well-posed extension of the minimal operator

$\prod_{j=1}^{n}\left[T_{0}\left(\tau_{j}\right)\right]$. For $f \in L_{w}^{2}(a, b)$ we put

$\varphi(t, \lambda)=R_{\lambda} f(t)$ then $\left[\prod_{j=1}^{n}\left(\tau_{j}\right)-\lambda I\right] \varphi=w f$ and consequently has a solution $\varphi(t, \lambda)$ in the form,

$$
\begin{aligned}
\varphi(t, \lambda)= & \sum_{j=1}^{n^{2} N} \alpha_{j}(\lambda) \varphi_{j}\left(t, \lambda_{0}\right)+\frac{1}{i^{n^{2} N}}\left(\lambda-\lambda_{0}\right) \\
& \sum_{j, k=1}^{n^{2} N} \xi^{j k} \varphi_{j}\left(t, \lambda_{0}\right) \int_{a}^{t} \frac{\varphi_{k}^{+}\left(t, \lambda_{0}\right)}{f}(s) w(s) \mathrm{d} s,
\end{aligned}
$$

for some constants $\alpha_{1}(\lambda), \alpha_{2}(\lambda), \cdots, \alpha_{n^{2} N}(\lambda) \in \mathbb{C} \quad$ (see Lemma 4.5). Since $f \in L_{w}^{2}(a, b)$ and $\varphi_{k}^{+}\left(., \lambda_{0}\right) \in L_{w}^{2}(a, b)$ for some $\lambda_{0} \in \mathbb{C}$, then $\varphi_{k}^{+}\left(., \lambda_{0}\right) f \in L_{w}^{1}(a, b), \quad k=1, \cdots, n^{2} N$ for some $\lambda_{0} \in \mathbb{C}$ and hence the integral in the right-hand of (6.4) will be finite.

To determine the constants $\alpha_{j}(\lambda), j=1, \cdots, n^{2} N$, let $\varphi_{k}^{+}(t, \lambda), k=1, \cdots, n^{2} N$ be a basis for $\left\{D\left(S^{*}\right) / D_{0}\left[\prod_{j=1}^{n}\left(\tau_{j}^{+}\right)\right]\right\}$, then because $\varphi(t, \lambda) \in D(S) \subset \rho(S) \subset \Delta_{4}(S)$, we have from Theorem 4.1 that,

$$
\begin{aligned}
& {\left[\varphi, \varphi_{k}^{+}\right](b)-\left[\varphi, \varphi_{k}^{+}\right](a)=0,} \\
& \left(k=1,2, \cdots, n^{2} N\right) \text { on }[a, b)
\end{aligned}
$$


and hence from (6.4), (6.5) and on using Lemma 4.6, we have:

$$
\begin{aligned}
& {\left[\varphi, \varphi_{k}^{+}\right](b)=\sum_{j=1}^{n^{2} N}\left[\alpha_{j}(\lambda)+\frac{1}{i^{n^{2} N}}\left(\lambda-\lambda_{0}\right) \times \sum_{j, k=1}^{n^{2} N} \xi^{j k} \int_{a}^{t} \overline{\varphi_{k}^{+}\left(t, \lambda_{0}\right)} f(s) w(s) \mathrm{d} s\right]\left[\varphi_{j}, \varphi_{k}^{+}\right](b),} \\
& {\left[\varphi, \varphi_{k}^{+}\right](a)=\sum_{j=1}^{n^{2} N} \alpha_{j}(\lambda)\left[\varphi_{j}, \varphi_{k}^{+}\right](a), \quad k=1,2, \cdots, n^{2} N .}
\end{aligned}
$$

By substituting these expressions into the conditions (6.5), we get:

$$
\begin{aligned}
& \sum_{j=1}^{n^{2} N}\left(\alpha_{j}(\lambda)+\frac{1}{i^{n^{2} N}}\left(\lambda-\lambda_{0}\right) \times \sum_{j, k=1}^{n^{2} N} \xi^{j k} \int_{a}^{t} \overline{\varphi_{k}^{+}\left(t, \lambda_{0}\right)} f(s) w(s) \mathrm{d} s\right)\left[\varphi_{j}, \varphi_{k}^{+}\right](b) \\
& =\sum_{j=1}^{n^{2} N} \alpha_{j}(\lambda)\left[\varphi_{j}, \varphi_{k}^{+}\right](a) .
\end{aligned}
$$

This implies that the system

$$
\sum_{j=1}^{n^{2} N} \alpha_{j}(\lambda)\left[\varphi_{j}, \varphi_{k}^{+}\right]_{a}^{b}=-\frac{\left(\lambda-\lambda_{0}\right)}{i^{n^{2} N}}\left(\sum_{j, k=1}^{n^{2} N} \xi^{j k} \int_{a}^{t} \overline{\varphi_{k}^{+}\left(t, \lambda_{0}\right)} f(s) w(s) \mathrm{d} s\right)
$$

in the variable $\alpha_{j}(\lambda), j=1,2, \cdots, n^{2} N$. The determinant of this system does not vanish (see [16, Theorem 3.27] and [19]). If we solve the system (6.7) we obtain:

$$
\alpha_{j}(\lambda)=\frac{\left(\lambda-\lambda_{0}\right)}{i^{n^{2} N}}\left(\sum_{j, k=1}^{n^{2} N} \xi^{j k} \int_{a}^{b} h_{j}(s, \lambda) f(s) w(s) \mathrm{d} s\right), j=1,2, \cdots, n^{2} N .
$$

where $h_{j}(s, \lambda)$ is a solution of the system:

$$
\sum_{j=1}^{n^{2} N} h_{j}(s, \lambda)\left(\left[\varphi_{j}, \varphi_{k}^{+}\right]\right)_{a}^{b}=-\sum_{j, k=1}^{n^{2} N} \xi^{j k} \overline{\varphi_{k}^{+}\left(t, \lambda_{0}\right)}\left[\varphi_{j}, \varphi_{k}^{+}\right](b)
$$

Since, the determinant of the above system (6.9) does not vanish, and the functions $\varphi_{k}^{+}\left(s, \lambda_{0}\right)$, $k=1,2, \cdots, n^{2} N$ are continuous in the interval $[a, b)$, then the functions $h_{j}(s, \lambda)$ are also continuous in the interval. By substituting in formula (6.4) for the expressions $\alpha_{j}(\lambda), j=1,2, \cdots, n^{2} N$ we get,

$$
\begin{aligned}
& R_{\lambda} f(t) \\
& =\varphi(t, \lambda)=\frac{\left(\lambda-\lambda_{0}\right)}{i^{n^{2} N}}\left[\sum_{j, k=1}^{n^{2} N} \alpha_{j}(\lambda) \varphi_{j}\left(t, \lambda_{0}\right)\right. \\
& \int_{a}^{t}\left[\xi^{j k} \overline{\varphi_{k}^{+}\left(t, \lambda_{0}\right)}+h_{j}(s, \lambda)\right] f(s) w(s) \mathrm{d} s \\
& \left.+\left(\sum_{j=1}^{n^{2} N} \varphi_{j}\left(t, \lambda_{0}\right) \int_{t}^{b} h_{j}(s, \lambda) f(s) w(s) \mathrm{d} s\right)\right]
\end{aligned}
$$

Now, we put

$$
\begin{aligned}
& K(t, s, \lambda) \\
& =\left\{\begin{array}{l}
\frac{\left(\lambda-\lambda_{0}\right)}{i^{n^{2} N}}\left(\sum_{j=1}^{n^{2} N} \varphi_{j}\left(t, \lambda_{0}\right) h_{j}(s, \lambda)\right) \\
\text { for } t<s \\
\frac{\left(\lambda-\lambda_{0}\right)}{i^{n^{2} N}}\left(\sum_{j, k=1}^{n^{2} N} \xi^{j k} \varphi_{j}\left(t, \lambda_{0}\right)\left(\overline{\varphi_{k}^{+}\left(t, \lambda_{0}\right)}+h_{j}(s, \lambda)\right)\right) \\
\text { for } t>s,
\end{array}\right.
\end{aligned}
$$

Formula (6.10) then takes the form

$$
\begin{aligned}
& R_{\lambda} f(t)=\int_{a}^{b} K(t, s, \lambda) f(s) w(s) \mathrm{d} s \\
& \text { for all } t \in[a, b),
\end{aligned}
$$

i.e., $R_{\lambda}$ is an integral operator with the kernel $K(s, t, \lambda)$ operating on the functions $f \in L_{w}^{2}(a, b)$. Similarly, the solutions $\varphi^{+}(t, \lambda)$ of the equation

$$
\begin{aligned}
& {\left[\prod_{j=1}^{n}\left(\tau_{j}^{+}\right)-\bar{\lambda} I\right] \varphi^{+}=w g \text { has the form: }} \\
& \quad \varphi^{+}(s, \lambda) \\
& \quad=\sum_{j=1}^{n^{2} N} \alpha_{j}(\lambda) \varphi_{j}^{+}\left(s, \lambda_{0}\right) \\
& \quad+\frac{\left(\bar{\lambda}-\bar{\lambda}_{0}\right)}{i^{n^{2} N}} \sum_{j, k=1}^{n^{2} N} \xi^{j k} \varphi_{j}^{+}\left(s, \lambda_{0}\right) \int_{a}^{s} \overline{\varphi_{k}\left(t, \lambda_{0}\right)} g(t) w(t) \mathrm{d} t,
\end{aligned}
$$

where $\varphi_{k}\left(t, \lambda_{0}\right)$ and $\varphi_{j}^{+}\left(s, \lambda_{0}\right), k, j=1,2, \cdots, n^{2} N$ are solutions of the equations in (6.6). The argument as before leads to,

$$
R_{\bar{\lambda}}^{*} g(t)=\int_{a}^{b} \overline{K^{+}(s, t, \bar{\lambda})} g(t) w(t) \mathrm{d} t \quad \text { for } g \in L_{w}^{2}(a, b),
$$

i.e., $R_{\bar{\lambda}}^{*}$ is an integral operator with the kernel $K^{+}(s, t, \bar{\lambda})$ operating on the functions $g \in L_{w}^{2}(a, b)$ where, 


$$
\begin{aligned}
& K^{+}(s, t, \bar{\lambda}) \\
& =\left\{\begin{array}{l}
\frac{\left(\bar{\lambda}-\bar{\lambda}_{0}\right)}{i^{n^{2} N}}\left(\sum_{j=1}^{n^{2} N} \varphi_{j}^{+}\left(s, \lambda_{0}\right) h_{j}^{+}(t, \lambda)\right) \\
\text { for } s<t \\
\frac{\left(\bar{\lambda}-\bar{\lambda}_{0}\right)}{i^{n^{2} N}}\left(\sum_{j, k=1}^{n^{2} N} \xi^{j k} \varphi_{j}^{+}\left(s, \lambda_{0}\right)\left(\overline{\varphi_{k}\left(t, \lambda_{0}\right)}+h_{j}^{+}(t, \lambda)\right)\right) \\
\text { for } s>t,
\end{array}\right.
\end{aligned}
$$

and $h_{j}^{+}(t, \lambda)$ is a solution of the system

$$
\begin{aligned}
& \sum_{j=1}^{n^{2} N} \overline{h_{j}(s, \lambda)}\left(\left[\varphi_{j}, \varphi_{k}^{+}\right]\right)_{a}^{b} \\
& =-\sum_{j, k=1}^{n^{2} N} \xi^{j k} \varphi_{j}\left(t, \lambda_{0}\right)\left[\varphi_{j}, \varphi_{k}^{+}\right](b) .
\end{aligned}
$$

From definitions of $R_{\lambda}$ and $R_{\bar{\lambda}}^{*}$, it follows that

$$
\begin{aligned}
\left(R_{\lambda} f, g\right) & =\int_{a}^{b}\left\{\int_{a}^{b} K(t, s, \lambda) f(s) w(s) \mathrm{d} s\right\} \overline{g(t)} w(t) \mathrm{d} t \\
& =\int_{a}^{b}\left\{\int_{a}^{b} K(t, s, \lambda) \overline{g(t)} w(t) \mathrm{d} t\right\} f(s) w(s) \mathrm{d} s \\
& =\left(f, R_{\bar{\lambda}}^{*} g\right),
\end{aligned}
$$

for any continuous functions $f, g \in H$ and by construction (see (6.11) and (6.15)), $K(t, s, \lambda)$ and $K^{+}(s, t, \bar{\lambda})$ are continuous functions on $[a, b) \times[a, b)$ and (6.17) gives us

$$
K(t, s, \lambda)=\overline{K^{+}(s, t, \bar{\lambda})} \text { for all } t, s \in[a, b) \times[a, b) .
$$

Since $\varphi_{j}(t, \lambda), \varphi_{k}^{+}(s, \lambda) \in L_{w}^{2}(a, b)$ for $j, k=1,2, \cdots, n^{2} N$ and for fixed $s, K(t, s, \lambda)$ is a linear combination of $\varphi_{j}(t, \lambda)$ while, for fixed $t$,

$K^{+}(s, t, \bar{\lambda})$ is a linear combination of $\varphi_{k}^{+}(s, \lambda)$. Then we have

$$
\begin{aligned}
& \int_{a}^{b}|K(t, s, \lambda)|^{2} w(t) \mathrm{d} t<\infty, \\
& \int_{a}^{b}\left|K^{+}(s, t, \bar{\lambda})\right|^{2} w(s) \mathrm{d} s<\infty, \quad a<s, t<b,
\end{aligned}
$$

and (6.18) implies that,

$$
\begin{aligned}
& \int_{a}^{b}|K(t, s, \lambda)|^{2} w(s) \mathrm{d} s=\int_{a}^{b}\left|K^{+}(s, t, \bar{\lambda})\right|^{2} w(s) \mathrm{d} s<\infty, \\
& \int_{a}^{b}\left|K^{+}(s, t, \bar{\lambda})\right|^{2} w(t) \mathrm{d} t=\int_{a}^{b}|K(t, s, \lambda)|^{2} w(t) \mathrm{d} t<\infty .
\end{aligned}
$$

Now, it is clear from (6.9) that the functions $h_{j}(s, \lambda),\left(j=1,2, \cdots, n^{2} N\right)$ belong to $L_{w}^{2}(a, b)$ since $h_{j}(s, \lambda)$ is a linear combination of the functions $\varphi_{j}^{+}(s, \lambda)$ which lie in $L_{w}^{2}(a, b)$ and hence $h_{j}(t, \lambda)$ belong to $L_{w}^{2}(a, b)$. Similarly $h_{j}^{+}(t, \lambda)$ belong to $L_{w}^{2}(a, b)$. By the upper half of the formula (6.11) and (6.15), we have:

$$
\int_{a}^{b}\left(\int_{a}^{b}|K(t, s, \lambda)|^{2} w(s) \mathrm{d} s\right) w(t) \mathrm{d} t<\infty,
$$

for the inner integral exists and is a linear combination of the products $\varphi_{j}(t, \lambda) \varphi_{k}^{+}(s, \lambda), \quad\left(j, k=1,2, \cdots, n^{2} N\right)$ and these products are integrable because each of the factors belongs to $L_{w}^{2}(a, b)$. Then by (6.18), and by the upper half of (6.15),

$$
\begin{aligned}
& \int_{a}^{b}\left(\int_{a}^{b}|K(t, s, \lambda)|^{2} w(s) \mathrm{d} s\right) w(t) \mathrm{d} t \\
& =\int_{a}^{b}\left(\int_{a}^{b}\left|K^{+}(s, t, \bar{\lambda})\right|^{2} w(s) \mathrm{d} s\right) w(t) \mathrm{d} t<\infty .
\end{aligned}
$$

Hence, we also have:

$$
\int_{a}^{b} \int_{a}^{b}|K(t, s, \lambda)|^{2} w(t) w(s) \mathrm{d} t \mathrm{~d} s<\infty,
$$

and the theorem is completely proved for any well-posed extension.

Remark 6.2: It follows immediately from Theorem 6.1 that, if for an operator $\prod_{j=1}^{n} T_{0}\left(\tau_{j}\right)$ with one singular end-point that

$$
\begin{aligned}
& \operatorname{def}\left[\prod_{j=1}^{n}\left[T_{0}\left(\tau_{j}\right)\right]-\lambda I\right] \\
& =\operatorname{def}\left[\prod_{j=1}^{n}\left[T_{0}\left(\tau_{j}^{+}\right)\right]-\bar{\lambda} I\right]=n^{2} N
\end{aligned}
$$

for all $\lambda \in \Pi\left[\prod_{j=1}^{n}\left[T_{0}\left(\tau_{j}\right)\right], \prod_{j=1}^{n}\left[T_{0}\left(\tau_{j}^{+}\right)\right]\right]$and $S$ is well-posed with respect to $\prod_{j=1}^{n} T_{0}\left(\tau_{j}\right)$ and $\prod_{j=1}^{n} T_{0}\left(\tau_{j}^{+}\right)$with $\lambda \in \rho(S)$ then $R_{\lambda}=(S-\lambda I)^{-1}$ is a Hilbert-Schmidt integral operator. Thus it is a completely continuous operator, and consequently its spectrum is discrete and consists of isolated eigenvalues having finite algebraic (so geometric) multiplicity with zero as the only possible point of accumulation. Hence, the spectra of all well-posed operators $S$ are discrete, i.e.,

$$
\sigma_{e k}(S)=\varnothing, \quad \text { for } k=1,2,3,4,5 .
$$

We refer to [6,7,11, Theorem IX.3.1], [15], [16] and [18] and for more details.

\section{The Case of Two Singular End-Points}

For the case of two singular end-points, we consider our interval to be $I=(a, b)$ and denote by $\prod_{j=1}^{n} T_{0}\left(\tau_{j}\right)$ and $\prod_{j=1}^{n} T\left(\tau_{j}\right)$ the product of minimal and maximal operators. We see from (3.15) and Lemma 4.2 that $\prod_{j=1}^{n}\left[T_{0}\left(\tau_{j}\right)\right] \subset \prod_{j=1}^{n}\left[T\left(\tau_{j}\right)\right]=\prod_{j=1}^{n}\left[T_{0}\left(\tau_{j}^{+}\right)\right]^{*}$ and hence $\prod_{j=1}^{n} T_{0}\left(\tau_{j}\right)$ and $\prod_{j=1}^{n} T_{0}\left(\tau_{j}^{+}\right)$form an adjoint pair of closed densely-defined operators in $L_{w}^{2}(a, b)$. 
For $\lambda \in \Pi\left[\prod_{j=1}^{n}\left[T_{0}\left(\tau_{j}\right)\right], \prod_{j=1}^{n}\left[T_{0}\left(\tau_{j}^{+}\right)\right]\right]$we define $r, s$ and $m$ as follows:

$$
\begin{aligned}
r= & r(\lambda):=\operatorname{def}\left[\prod_{j=1}^{n} T_{0}\left(\tau_{j}\right)-\lambda I\right] \\
= & \operatorname{def}\left[\prod_{j=1}^{n} T_{0}\left(\tau_{j} ; a\right)-\lambda I\right] \\
& +\operatorname{def}\left[\prod_{j=1}^{n} T_{0}\left(\tau_{j} ; b\right)-\lambda I\right]-n^{2} N \\
= & r_{1}+r_{2}-n^{2} N \\
s= & s(\lambda):=\operatorname{def}\left[\prod_{j=1}^{n} T_{0}\left(\tau_{j}^{+}\right)-\bar{\lambda} I\right] \\
= & \operatorname{def}\left[\prod_{j=1}^{n} T_{0}\left(\tau_{j}^{+} ; a\right)-\bar{\lambda} I\right] \\
+ & \operatorname{def}\left[\prod_{j=1}^{n} T_{0}\left(\tau_{j}^{+} ; b\right)-\bar{\lambda} I\right]-n^{2} N \\
= & s_{1}+s_{2}-n^{2} N,
\end{aligned}
$$

and

$$
\begin{aligned}
m & =r+s=\left(r_{1}+r_{2}-n^{2} N\right)+\left(s_{1}+s_{2}-n^{2} N\right) \\
& =\left(r_{1}+s_{1}\right)+\left(r_{2}+s_{2}\right)-2 n^{2} N \\
& =\left(m_{1}+m_{2}\right)-2 n^{2} N
\end{aligned}
$$

Also, since $n^{2} N \leq m_{i} \leq 2 n^{2} N(i=1,2)$, then by Lemma 5.2 we have that, $0 \leq m \leq 2 n^{2} N$.

For an operator $\prod_{j=1}^{n} T_{0}\left(\tau_{j}\right)$ with two singular end-points, Theorem 6.1 remains true in its entirely, that is all well-posed extensions of the minimal operator $\prod_{j=1}^{n} T_{0}\left(\tau_{j}\right)$ in the maximal case, i.e., when $r_{1}=r_{2}=n^{2} N$ and $s_{1}=s_{2}=n^{2} N$ in (7.1) and (7.2) have resolvents which are Hilbert-Schmidt integral operators and consequently have a wholly spectrum, and hence Remark 6.2 also remains valid. This implies as in Corollary 7.2 below that all the regularly solvable operators have standard essential spectra to be empty. We refer to $[1,2,6,7,10,11,15]$ and $[16]$ for more details.

Now, we prove Theorem 6.1 in the case of two singular end-points.

Theorem 7.1: Suppose for an operator $\prod_{j=1}^{n} T_{0}\left(\tau_{j}\right)$ with two singular end-points that,

$$
\begin{aligned}
& \operatorname{def}\left[\prod_{j=1}^{n} T_{0}\left(\tau_{j}\right)-\lambda I\right] \\
& =\operatorname{def}\left[\prod_{j=1}^{n} T_{0}\left(\tau_{j}^{+}\right)-\bar{\lambda} I\right]=n^{2} N
\end{aligned}
$$

for all $\lambda \in \Pi\left[\prod_{j=1}^{n}\left[T_{0}\left(\tau_{j}\right)\right], \prod_{j=1}^{n}\left[T_{0}\left(\tau_{j}^{+}\right)\right]\right]$and let $S$ be an arbitrary closed operator which is a well-posed extension of the minimal operator $\prod_{j=1}^{n} T_{0}\left(\tau_{j}\right)$ and $\lambda \in \rho(S)$, then the resolvent $R_{\lambda}$ and $R_{\bar{\lambda}}^{*}$ of $S$ and $S^{*}$ respectively are Hilbert-Schmidt integral operators whose kernels are continuous functions on $[a, b) \times[a, b)$ and satisfy (6.2).

Proof: Let,

$$
\begin{aligned}
& \operatorname{def}\left[\prod_{j=1}^{n}\left[T_{0}\left(\tau_{j}\right)\right]-\lambda I\right] \\
& =\operatorname{def}\left[\prod_{j=1}^{n}\left[T_{0}\left(\tau_{j}^{+}\right)\right]-\bar{\lambda} I\right]=n^{2} N
\end{aligned}
$$

for all $\lambda \in \Pi\left[\prod_{j=1}^{n}\left[T_{0}\left(\tau_{j}\right)\right], \prod_{j=1}^{n}\left[T_{0}\left(\tau_{j}^{+}\right)\right]\right]$, then we choose a fundamental system of solutions

$$
\begin{array}{r}
\Phi_{j}(t, \lambda) \text { and } \psi_{j}(t, \lambda),\left(j=1,2, \cdots, n^{2} N\right) \text { as: } \\
\Phi_{j}(t, \lambda)= \begin{cases}\Phi_{j}^{a}(t, \lambda) & \text { on }(a, c] \\
\Phi_{j}^{b}(t, \lambda) & \text { on }[c, b)\end{cases} \\
\psi_{j}(t, \lambda)= \begin{cases}\psi_{j}^{a}(t, \lambda) & \text { on }(a, c] \\
\psi_{j}^{b}(t, \lambda) & \text { on }[c, b)\end{cases}
\end{array}
$$

of the equations in (6.3), so that $\Phi_{j}(t, \lambda)$ and $\psi_{j}(t, \lambda),\left(j=1,2, \cdots, n^{2} N\right)$ belong to $L_{w}^{2}(a, b)$, i.e., they are quadratically integrable in the interval $(a, b)$.

Let $R_{\lambda}=(S-\lambda I)^{-1}$ be the resolvent of any wellposed extension $S=S^{a} \oplus S^{b}$ of the minimal operator $\prod_{j=1}^{n} T_{0}\left(\tau_{j}\right)$. For $f \in\left[L_{w}^{2}(a, c) \oplus L_{w}^{2}(c, b)\right]$ we put $\varphi(t, \lambda)=R_{\lambda} f(t)$, then $\left[\prod_{j=1}^{n}\left(\tau_{j}\right)-\lambda I\right] \Phi=w f \quad$ and hence as in (6.4) we have,

$$
\begin{aligned}
& R_{\lambda} f(t)=\Phi(t, \lambda)=\sum_{j=1}^{n^{2} N} \alpha_{j}(\lambda) \Phi_{j}\left(t, \lambda_{0}\right)+\frac{1}{i^{n^{2} N}}\left(\lambda-\lambda_{0}\right) \\
& \times \sum_{j, k=1}^{n^{2} N} \xi^{j k} \Phi_{j}\left(t, \lambda_{0}\right) \int_{a}^{t} \overline{\Phi_{k}^{+}\left(t, \lambda_{0}\right)} f(s) w(s) \mathrm{d} s,
\end{aligned}
$$

for some constants $\alpha_{1}(\lambda), \alpha_{2}(\lambda), \cdots, \alpha_{n^{2} N}(\lambda) \in \mathbb{C}$ where,

$$
\Phi(t, \lambda)=\left\{\begin{array}{ll}
\Phi^{a}(t, \lambda) & \text { on }(a, c] \\
\Phi^{b}(t, \lambda) & \text { on }[c, b)
\end{array} .\right.
$$

By proceeding as in Theorem 6.1, we get $\alpha_{j}(\lambda)$ as in (6.8),

$$
\begin{aligned}
& \alpha_{j}(\lambda)=\frac{\left(\lambda-\lambda_{0}\right)}{i^{n^{2} N}}\left(\sum_{j, k=1}^{n^{2} N} \xi^{j k} \int_{a}^{b} h_{j}(s, \lambda) f(s) w(s) \mathrm{d} s\right), \\
& j=1,2, \cdots, n^{2} N
\end{aligned}
$$

where

$$
h_{j}(t, \lambda)=\left\{\begin{array}{lc}
h_{j}^{a}(t, \lambda) & \text { on }(a, c] \\
h_{j}^{b}(t, \lambda) & \text { on }[c, b)
\end{array}, \quad j=1,2, \cdots, n^{2} N .\right.
$$

By substituting in (7.6) for the constants $\alpha_{j}(\lambda)$, $j=1,2, \cdots, n^{2} N$ we get,

$$
R_{\lambda} f(t)=\int_{a}^{b} K(t, s, \lambda) f(s) w(s) \mathrm{d} s \quad \text { for all } t \in[a, b),
$$




$$
K(t, s, \lambda)=\left\{\begin{array}{lc}
K^{a}(t, s, \lambda) & \text { on }(a, c] \\
K^{b}(t, s, \lambda) & \text { on }[c, b)
\end{array},\right.
$$

and $K^{(.)}(t, s, \lambda)$ can be obtained as in (6.11). Similarly,

$$
\begin{array}{rr}
R_{\bar{\lambda}}^{*} g(t)=\int_{a}^{b} K^{+}(s, t, \bar{\lambda}) g(s) w(s) \mathrm{d} s & \text { for } g \in L_{w}^{2}(a, b), \\
K^{+}(s, t, \bar{\lambda})= \begin{cases}K^{+(a)}(s, t, \bar{\lambda}) & \text { on }(a, c] \\
K^{+(b)}(s, t, \bar{\lambda}) & \text { on }[c, b)\end{cases}
\end{array}
$$

From (6.11) and (6.15) we have that

$$
\begin{aligned}
& \int_{a}^{b}|K(t, s, \lambda)|^{2} w(t) \mathrm{d} t<\infty, \\
& \int_{a}^{b}\left|K^{+}(s, t, \bar{\lambda})\right|^{2} w(s) \mathrm{d} s<\infty, \quad a<s, t<b,
\end{aligned}
$$

and (6.18) implies that,

$$
\begin{gathered}
\int_{a}^{b}|K(t, s, \lambda)|^{2} w(s) \mathrm{d} s=\int_{a}^{b}\left|K^{+}(s, t, \bar{\lambda})\right|^{2} w(s) \mathrm{d} s<\infty \\
\int_{a}^{b}\left|K^{+}(s, t, \bar{\lambda})\right|^{2} w(t) \mathrm{d} t=\int_{a}^{b}|K(t, s, \lambda)|^{2} w(t) \mathrm{d} t<\infty
\end{gathered}
$$

The rest of the proof is entirely similar to the corresponding part of the proof of Theorem 6.1. We refer to [1, $5,6,7,15,16]$ and [18] for more details.

Corollary 7.2: Let $\lambda \in \Pi\left[\prod_{j=1}^{n} T_{0}\left(\tau_{j}\right), \prod_{j=1}^{n} T_{0}\left(\tau_{j}^{+}\right)\right]$ with

$$
\begin{aligned}
& \operatorname{def}\left[\prod_{j=1}^{n}\left[T_{0}\left(\tau_{j}\right)\right]-\lambda I\right] \\
& =\operatorname{def}\left[\prod_{j=1}^{n}\left[T_{0}\left(\tau_{j}^{+}\right)\right]-\bar{\lambda} I\right]=n^{2} N .
\end{aligned}
$$

Then,

$$
\sigma_{e k}(S)=\varnothing, \quad \text { for } k=1,2,3 .
$$

of all regularly solvable extensions $S$ with respect to the compatible adjoint pair $\prod_{j=1}^{n} T_{0}\left(\tau_{j}\right)$ and

$$
\prod_{j=1}^{n} T_{0}\left(\tau_{j}^{+}\right)
$$

\section{Proof: Since}

$$
\begin{aligned}
& \operatorname{def}\left[\prod_{j=1}^{n}\left[T_{0}\left(\tau_{j}\right)\right]-\lambda I\right] \\
& =\operatorname{def}\left[\prod_{j=1}^{n}\left[T_{0}\left(\tau_{j}^{+}\right)\right]-\bar{\lambda} I\right]=n^{2} N,
\end{aligned}
$$

for all $\lambda \in \Pi\left[\prod_{j=1}^{n}\left[T_{0}\left(\tau_{j}\right)\right], \prod_{j=1}^{n}\left[T_{0}\left(\tau_{j}^{+}\right)\right]\right]$. Then we have from [15, Theorem III.3.5] that,

$$
\begin{aligned}
& \operatorname{dim}\left\{D(S) / D_{0}\left[\prod_{j=1}^{n} T_{0}\left(\tau_{j}\right)\right]\right\} \\
& =\operatorname{def}\left[\prod_{j=1}^{n} T_{0}\left(\tau_{j}\right)-\lambda I\right]=n^{2} N,
\end{aligned}
$$

$$
\begin{aligned}
& \operatorname{dim}\left\{D\left(S^{*}\right) / D_{0}\left[\prod_{j=1}^{n} T_{0}\left(\tau_{j}^{+}\right)\right]\right\} \\
& =\operatorname{def}\left[\prod_{j=1}^{n} T_{0}\left(\tau_{j}^{+}\right)-\bar{\lambda} I\right]=n^{2} N .
\end{aligned}
$$

Thus $S$ is an $n^{2} N$-dimensional extension of $\prod_{j=1}^{n} T_{0}\left(\tau_{j}\right)$ and so by [11, Corollary IX.4.2],

$$
\sigma_{e k}(S)=\sigma_{e k}\left[\prod_{j=1}^{n} T_{0}\left(\tau_{j}\right)\right], \quad(k=1,2,3) .
$$

From Lemmas 4.12 and 4.13, we get,

$$
\sigma_{e k}\left[\prod_{j=1}^{n} T_{0}\left(\tau_{j}\right)\right]=\varnothing, \quad(k=1,2,3) .
$$

Hence, by (7.14) we have that,

$$
\sigma_{e k}(S)=\varnothing, \quad(k=1,2,3) \text {. }
$$

Remark 7.3: If $S$ is well-posed (say the Visik extension, see [20]) we get from (6.19) and (7.13) that

$$
\sigma_{e k}\left[\prod_{j=1}^{n} T_{0}\left(\tau_{j}\right)\right], \quad(k=1,2,3) .
$$

On applying (7.13) again to any regularly solvable extensions $S$ under consideration, hence (7.12).

Corollary 7.4: If for some $\lambda_{0} \in \mathbb{C}$, there are $n^{2} N$ linearly independent solutions of the equations

$$
\left[\prod_{j=1}^{n} \tau_{j}-\lambda_{0} w\right] u=0, \quad\left(\prod_{j=1}^{n} \tau_{j}^{+}-\bar{\lambda}_{0} w\right) v=0
$$

in $L_{w}^{2}(a, b), \lambda_{0} \in \Pi\left[\prod_{j=1}^{n} T_{0}\left(\tau_{j}\right), \prod_{j=1}^{n} T_{0}\left(\tau_{j}^{+}\right)\right]$and hence,

$$
\begin{aligned}
& \Pi\left[\prod_{j=1}^{n} T_{0}\left(\tau_{j}\right), \prod_{j=1}^{n} T_{0}\left(\tau_{j}^{+}\right)\right]=\mathbb{C} \text { and } \\
& \sigma_{e k}\left[\prod_{j=1}^{n} T_{0}\left(\tau_{j}\right), \prod_{j=1}^{n} T_{0}\left(\tau_{j}^{+}\right)\right]=\varnothing, \quad k=1,2,3,
\end{aligned}
$$

where $\sigma_{e k}\left[\prod_{j=1}^{n} T_{0}\left(\tau_{j}\right), \prod_{j=1}^{n} T_{0}\left(\tau_{j}^{+}\right)\right]$is the joint essential spectra of $\prod_{j=1}^{n} T_{0}\left(\tau_{j}\right), \quad \prod_{j=1}^{n} T_{0}\left(\tau_{j}^{+}\right)$defined as the joint field of regularity

$$
\Pi\left[\prod_{j=1}^{n} T_{0}\left(\tau_{j}\right), \prod_{j=1}^{n} T_{0}\left(\tau_{j}^{+}\right)\right] .
$$

Proof: Since all solutions of the equations in (7.15) are in $L_{w}^{2}(a, b)$ for some $\lambda_{0} \in \mathbb{C}$ in then,

$$
\begin{aligned}
& \operatorname{def}\left[\prod_{j=1}^{n} T_{0}\left(\tau_{j}\right)-\lambda_{0} I\right] \\
& =\operatorname{def}\left[\prod_{j=1}^{n} T_{0}\left(\tau_{j}^{+}\right)-\bar{\lambda}_{0} I\right]=n^{2} N,
\end{aligned}
$$

for some $\lambda_{0} \in \Pi\left[\prod_{j=1}^{n} T_{0}\left(\tau_{j}\right), \prod_{j=1}^{n} T_{0}\left(\tau_{j}^{+}\right)\right]$. From Lemma 3.10, we have that $\prod_{j=1}^{n} T_{0}\left(\tau_{j}\right)$ has no eigenvalues and so $\left[\prod_{j=1}^{n} T_{0}\left(\tau_{j}\right)-\lambda_{0} I\right]^{-1}$ exists and its domain $R\left[\prod_{j=1}^{n} T_{0}\left(\tau_{j}\right)-\lambda_{0} I\right]$ is a closed subspace of $L_{w}^{2}(a, b)$. 
Hence, since $\prod_{j=1}^{n} T_{0}\left(\tau_{j}\right)$ is a closed operator, then $\left[\prod_{j=1}^{n} T_{0}\left(\tau_{j}\right)-\lambda_{0} I\right]^{-1}$ is bounded and hence $\Pi\left[\prod_{j=1}^{n} T_{0}\left(\tau_{j}\right)\right]=\mathbb{C}$. Similarly $\Pi\left[\prod_{j=1}^{n} T_{0}\left(\tau_{j}^{+}\right)\right]=\mathbb{C}$. Therefore $\Pi\left[\prod_{j=1}^{n} T_{0}\left(\tau_{j}\right), \prod_{j=1}^{n} T_{0}\left(\tau_{j}^{+}\right)\right]=\mathbb{C}$ and hence,

$$
\begin{aligned}
& \operatorname{def}\left[\prod_{j=1}^{n} T_{0}\left(\tau_{j}\right)-\lambda I\right] \\
& =\operatorname{def}\left[\prod_{j=1}^{n} T_{0}\left(\tau_{j}^{+}\right)-\bar{\lambda} I\right]=n^{2} N
\end{aligned}
$$

for all $\lambda \in \Pi\left[\prod_{j=1}^{n} T_{0}\left(\tau_{j}\right), \prod_{j=1}^{n} T_{0}\left(\tau_{j}^{+}\right)\right]$. From Corollary 7.2 we have for any regularly solvable extension $S$ of $\prod_{j=1}^{n} T_{0}\left(\tau_{j}\right)$ that $\sigma_{e k}(S)=\varnothing, k=1,2,3$. and by (7.14) we get $\sigma_{e k}\left[\prod_{j=1}^{n} T_{0}\left(\tau_{j}\right)\right]=\varnothing, k=1,2,3$. Similarly $\sigma_{e k}\left[\prod_{j=1}^{n} T_{0}\left(\tau_{j}^{+}\right)\right]=\varnothing, k=1,2,3$. Hence,

$$
\sigma_{e k}\left[\prod_{j=1}^{n} T_{0}\left(\tau_{j}\right), \prod_{j=1}^{n} T_{0}\left(\tau_{j}^{+}\right)\right]=\varnothing, \quad k=1,2,3 .
$$

Remark 7.5: If there are $n^{2} N$ linearly independent solutions of the Equations (7.15) in $L_{w}^{2}(a, b)$ for some $\lambda_{0} \in \mathbb{C}$ then the complex plane can be divided into two disjoint sets:

$$
\begin{aligned}
\mathbb{C}= & \Pi\left[\prod_{j=1}^{n} T_{0}\left(\tau_{j}\right), \prod_{j=1}^{n} T_{0}\left(\tau_{j}^{+}\right)\right] \\
& \cup \sigma_{e k}\left[\prod_{j=1}^{n} T_{0}\left(\tau_{j}\right), \prod_{j=1}^{n} T_{0}\left(\tau_{j}^{+}\right)\right], \quad k=1,2,3 .
\end{aligned}
$$

We refer to $[6,7,10,16,18,19]$ for more details.

\section{REFERENCES}

[1] N. I. Akhiezer and I. M. Glazman, "Theory of Linear Operators in Hilbert Space," Frederich Unger Publishing Co., New York, 1963.

[2] M. N. Naimark, "Linear Differential Operators," New York, Ungar, Part I, 1967, Part II, 1968.

[3] W. N. Everitt and A. Zettl, "The Number of Integrable Square Solutions of Products of Differential Expressions," Proceedings of the Royal Society of Edinburgh, Edinburgh, Vol. 76 A, 1977, pp. 215-226.

[4] W. N. Everitt and A. Zettl, "Generalized Symmetric Ordinary Differential Expressions I," The General Theory. Niew Archief Voor Wiskunde, Vol. 1, No. 3, 1979, pp. 363-397.

[5] A. N. Krall and A. Zettl, "Singular Self-Adjoint SturmLiouville Problems," Journal of Differential and Integral Equations, Vol. 1, No. 4, 1998, pp. 423-432.

[6] D. Race, "On the Location of the Essential Spectra and Regularity Fields of Complex Sturm-Liouville Operators," Proceedings of the Royal Society of Edinburgh, Edinburgh, Vol. 85A, 1980, pp. 1-14.

\section{doi:10.1017/S0308210500011689}

[7] D. Race, "On the Essential Spectra of Linear 2nd Order Differential Operators with Complex Coefficients," Proceedings of the Royal Society of Edinburgh, Edinburgh, Vol. 92A, 1982, pp. 65-75. doi:10.1017/S0308210500019934

[8] A. Zettl, "Deficiency Indices of Polynomials in Symmetric Differential Expressions," II, Proceedings of the Royal Society of Edinburgh, Edinburgh, Vol. 73A, No. 20, 1974 (1975), pp. 301-306.

[9] A. Zettl, "Formally Self-Adjoint Quasi-Differential Operators," Rocky Mountain Journal of Mathematics, Vol. 5, No. 3, 1975, pp. 453-474. doi:10.1216/RMJ-1975-5-3-453

[10] S. E Ibrahim, "On the Products of Self-Adjoint SturmLiouville Differential Operators in Direct Sum Spaces," Journal of Informatics and Mathematical Sciences, Vol. 4 No. 1, 2012, pp. 93-109.

[11] D. E. Edmunds and W. D. Evans, "Spectral Theory and Differential Operators," Oxford University Press, Oxford, 1987.

[12] W. D. Evans, "Regularly Solvable Extensions of NonSelf-Adjoint Ordinary Differential Operators," Proceedings of the Royal Society of Edinburgh, Edinburgh, Vol. 114 A, 1990, pp. 99-117.

[13] W. D. Evans and S. E. Ibrahim, "Boundary Conditions for General Ordinary Differential Operators," Proceedings of the Royal Society of Edinburgh, Edinburgh, Vol. 97A 1984, pp. 79-95. doi:10.1017/S0308210500031851

[14] W. N. Everitt and D. Race, "Some Remarks on Linear Ordinary Quasi-Differential Expressions," Journal of London Mathematical Society, Vol. 3, No. 54, 1987, pp. 300320.

[15] S. E. Ibrahim, "Non-Self-Adjoint Quasi-Differential Operators with Discrete Spectra," Rocky Mountain Journal of Mathematics, Vol. 25, No. 3, 1995, pp. 1053-1348. doi: $10.1216 / \mathrm{rmjm} / 1181072204$

[16] S. E. Ibrahim, "The Spectra of Well-Posed Operators," Proceedings of the Royal Society of Edinburgh, Edinburgh, Vol. 125 A, 1995, pp. 1331-1348.

[17] S. E. Ibrahim, "The Point Spectra and Regularity Fields of Products of Quasi-Differential Operators," Indian Journal of Pure and Applied Mathematics, Vol. 31, No. 6, 2000, pp. 747-665.

[18] S. E. Ibrahim, "On the Essential Spectra of General Differential Operators," Italian Journal of Pure and Applied Mathematics Y, No. 9, 2001, pp. 45-67.

[19] S. E Ibrahim, "On the Essential Spectra for Products of the General Quasi-Differential Operators and Their Adjoints," International Journal of Pure and Applied Mathematics, Vol. 70, No. 5, 2011, pp. 659-689.

[20] M. I. Visik, "On General Boundary Problems for Elliptic Differential Equations," American Mathematical Society Transl, Vol. 2, No. 24, 1963, pp. 107-172.

[21] N. A. Zhikhar, "The Theory of Extension of J-Symmetric Operators," Ukranin, Mat. Z. XI, Vol. 4, 1959, pp. 352365. 